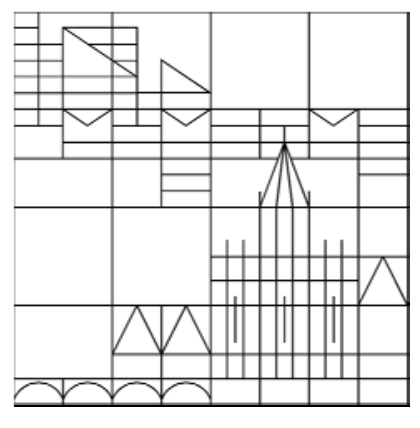

\title{
Analytic semigroups of pseudodifferential operators on vector-valued Sobolev spaces
}

\author{
Bienvenido Barraza Martinez \\ Robert Denk \\ Jairo Hernández Monzón
}

Konstanzer Schriften in Mathematik

Nr. 303, Mai 2012

ISSN 1430-3558 



\title{
ANALYTIC SEMIGROUPS OF PSEUDODIFFERENTIAL OPERATORS ON VECTOR-VALUED SOBOLEV SPACES
}

\author{
B. BARRAZA MARTINEZ, R. DENK, AND J. HERNÁNDEZ MONZÓN
}

\begin{abstract}
In this paper we study continuity and invertibility of pseudodifferential operators with non-regular Banach space valued symbols. The corresponding pseudodifferential operators generate analytic semigroups on the Sobolev spaces $W_{p}^{k}\left(\mathbb{R}^{n}, E\right)$ with $k \in \mathbb{N}_{0}, 1 \leq p \leq \infty$. Here $E$ is an arbitrary Banach space. We also apply the theory to solve nonautonomous parabolic pseudodifferential equations in Sobolev spaces.
\end{abstract}

\section{INTRODUCTION}

In the present work, we regard symbols in the space $S_{1,0}^{m, \rho}\left(\mathbb{R}^{n}, E\right)$ with $m \in \mathbb{R}, \rho \in \mathbb{N}_{0}$ and $E$ being an arbitrary Banach space. We say that $a \in$ $S_{1,0}^{m, \rho}\left(\mathbb{R}^{n}, E\right)$ if $a \in C^{\rho}\left(\mathbb{R}^{n}, E\right)$ and if for all $\alpha \in \mathbb{N}_{0}^{n}$ with $|\alpha| \leq \rho$ there exists a $c_{\alpha}>0$ such that

$$
\left\|\partial_{\xi}^{\alpha} a(\xi)\right\|_{E} \leq c_{\alpha}\langle\xi\rangle^{m-|\alpha|} \text { for all } \xi \in \mathbb{R}^{n} .
$$

Here $\langle\xi\rangle:=\sqrt{1+|\xi|^{2}}, \xi \in \mathbb{R}^{n}$. Because of Mikhlin's theorem (see [BL76] and [NNH02]) the pseudodifferential operator or the Fourier multiplier induced by $a$,

$$
\mathcal{F}^{-1} a \mathcal{F}: L_{p}\left(\mathbb{R}^{n}, E_{1}\right) \longrightarrow L_{p}\left(\mathbb{R}^{n}, E_{2}\right) \quad \text { for } 1<p<\infty,
$$

is continuous if $a \in S_{1,0}^{0, n+1}\left(\mathbb{R}^{n}, \mathcal{L}\left(E_{1}, E_{2}\right)\right)$ and $E_{1}, E_{2}$ are Hilbert spaces. Here $\mathcal{F}$ and $\mathcal{F}^{-1}$ denote the Fourier transform and the inverse Fourier transform, respectively. In practice one would like to have the validity of this result for arbitrary Banach spaces $E_{1}, E_{2}$. But, this is impossible in light of an observation of G. Pisier: If the Mikhlin theorem is valid on $L_{p}\left(\mathbb{R}^{n}, E\right)$ for $\mathcal{L}(E)$-vector-valued symbols, then $E$ is isomorphic to a Hilbert space (see [LLM98] for a proof).

Therefore, in order to obtain versions of Mikhlin's theorem, one has to, for example, change the space scale $L_{p}\left(\mathbb{R}^{n}, E\right)$, make additional conditions for (1), or impose conditions on the geometry of the Banach space $E$. On one hand, Amann has shown in [Am97], Theorem 6.2, that for arbitrary

B. Barraza Martinez, Universidad del Norte, Departamento de MATEMÁticas, BARRANQUiLla (COLOMBia)

R. Denk, Universität Konstanz, Fachbereich für Mathematik und Statistik, Konstanz (GERmany)

J. Hernández Monzón, Universidad del Norte, Departamento de Matemáticas, BarRanquilla (COlombia)

E-mail addresses: bbarraza@uninorte.edu.co, robert.denk@uni-konstanz.de, jahernan@uninorte.edu.co.

Date: May 8th, 2012. 
Banach spaces $E_{1}$ and $E_{2}$ and $a \in S_{1,0}^{m, n+1}\left(\mathbb{R}^{n}, \mathcal{L}\left(E_{1}, E_{2}\right)\right)$ that the pseudodifferential operator $\mathcal{F}^{-1} a \mathcal{F}: B_{p, q}^{s+m}\left(\mathbb{R}^{n}, E_{1}\right) \longrightarrow B_{p, q}^{s}\left(\mathbb{R}^{n}, E_{2}\right)(s \in \mathbb{R}$, $p, q \in[1, \infty])$ is continuous, where $B_{p, q}^{s}\left(\mathbb{R}^{n}, E\right)$ denotes the Besov space.

On the other hand, Weis proved in [We01] for UMD spaces $E_{1}$ and $E_{2}$ and symbols $a$ in $S_{1,0}^{0,1}\left(\mathbb{R}, \mathcal{L}\left(E_{1}, E_{2}\right)\right)$ which fulfill a stronger condition than (1), that $\mathcal{F}^{-1} a \mathcal{F}: L_{p}\left(\mathbb{R}, E_{1}\right) \longrightarrow L_{p}\left(\mathbb{R}, E_{2}\right)$ (for $1<p<\infty$ ) is continuous. Similar results were obtained in [PS06]. By a UMD space $E$ one understands a Banach space for which the Hilbert transform is continuous from $L_{p}(\mathbb{R}, E)$ to $L_{p}(\mathbb{R}, E)$ for $1<p<\infty$, or equivalently, for which the function $m(t)=|t|^{-1} t$ is a Fourier multiplier on $L_{p}(\mathbb{R}, E)(1<p<\infty)$. This implies reflexivity of $E$ (see [Am95], Remark 4.4.2).

In order to achieve an optimal value of $\rho$ in (1), one has to regard the geometry of the Banach spaces $E_{1}, E_{2}$. Girardi and Weis considered in [GW03] Banach spaces $E_{1}, E_{2}$ of Fourier type $p, 1 \leq p \leq 2$, (i.e. Banach spaces $E_{i}$ for which the Fourier transform is continuous of $L_{p}\left(\mathbb{R}^{n}, E_{i}\right)$ into $L_{p^{\prime}}\left(\mathbb{R}^{n}, E_{i}\right)$ with $\left.\frac{1}{p}+\frac{1}{p^{\prime}}=1\right)$, and symbols $a \in S_{1,0}^{0, \rho}\left(\mathbb{R}^{n}, \mathcal{L}\left(E_{1}, E_{2}\right)\right)$. Under these conditions, they proved that for all $s \in \mathbb{R}, r, q \in[1, \infty]$, the pseudodifferential operator $\mathcal{F}^{-1} a \mathcal{F}: B_{r, q}^{s}\left(\mathbb{R}^{n}, E_{1}\right) \longrightarrow B_{r, q}^{s}\left(\mathbb{R}^{n}, E_{2}\right)$ is continuous, if $\rho=\left[\frac{n}{p}\right]+1$. For for arbitrary Banach spaces $E_{1}, E_{2}$ the Fourier type equals $p=1$, and one obtains Theorem 6.2 in [Am97]. If $E_{1}, E_{2}$ are uniformly convex Banach spaces (thus having Fourier type $p>1$ ), see [Bo87], one can choose $\rho=n$.

Many applications to problems of physics and biology, e.g. models for reaction-diffusion processes (see $[\mathrm{Am00}]$ ), suggest the necessity to regard Banach space-valued symbols. The space considered in [Am00] is the vectorvalued Sobolev-Slobodeckii space $W_{1}^{s}\left(\mathbb{R}^{n}, L_{1}(Y, \mu)\right), s \notin \mathbb{N}$, and it is wellknown that $L_{1}(Y, \mu)$ is not a reflexive space. For this reason, we would like to obtain a version of the Mikhlin theorem (like in [Am97]) for Banach space-valued symbols, in which the correspondent pseudodifferential operator generates an analytic semigroup on $W_{p}^{k}\left(\mathbb{R}^{n}, E\right), 1 \leq p \leq \infty$. As a consequence, one obtains existence and uniqueness of solutions of parabolic pseudodifferential equations.

Therefore, we regard arbitrary Banach spaces $E_{i}, i=0,1,2$, the vector space

$$
\mathbf{V}\left(\mathbb{R}^{n}, E_{i}\right):=\bigcup_{(s, p, q) \in \mathbb{R} \times[1, \infty] \times[1, \infty]} B_{p, q}^{s}\left(\mathbb{R}^{n}, E_{i}\right)
$$

and symbols $a \in S_{1,0}^{m, \rho_{n}}\left(\mathbb{R}^{n}, E_{1}\right)$ with regularity

$$
\rho_{n}:=\left\{\begin{array}{l}
n+1, n \in \mathbb{N} \text { odd } \\
n+2, n \in \mathbb{N} \text { even }
\end{array}\right.
$$

We will show (see Theorem 3.1) that there exists a unique linear map

$$
\widetilde{a(D)}: \mathbf{V}\left(\mathbb{R}^{n}, E_{2}\right) \longrightarrow \mathbf{V}\left(\mathbb{R}^{n}, E_{0}\right)
$$

with $\widetilde{a(D)} \in \mathcal{L}\left(B_{p, q}^{s+m}\left(\mathbb{R}^{n}, E_{2}\right), B_{p, q}^{s}\left(\mathbb{R}^{n}, E_{0}\right)\right)$ such that its restriction on every intersection $C_{b}^{\infty}\left(\mathbb{R}^{n}, E_{2}\right) \cap B_{p, q}^{s+m}\left(\mathbb{R}^{n}, E_{2}\right)(s \in \mathbb{R}, p, q \in[1, \infty])$ coincides with the classical definition of the pseudodifferential operator $a(D)$ in [Ku81], which has been defined by means of an oscillatory integral. In 
this way, we show that suitable restrictions of the operator $-\widetilde{a(D)}$ generate analytic semigroups on the Besov spaces $B_{p, q}^{s}\left(\mathbb{R}^{n}, E_{0}\right)$ and $C^{\infty}$-semigroups on the Sobolev spaces $W_{p}^{k}\left(\mathbb{R}^{n}, E_{0}\right)$. Thus we obtain results similar to those in [Am97]: Using $\widetilde{a(D)} \in \mathcal{L}\left(B_{p, q}^{s+m}\left(\mathbb{R}^{n}, E_{2}\right), B_{p, q}^{s}\left(\mathbb{R}^{n}, E_{0}\right)\right)$, the continuous embedding

$$
B_{p, 1}^{k}\left(\mathbb{R}^{n}, E\right) \hookrightarrow W_{p}^{k}\left(\mathbb{R}^{n}, E\right) \hookrightarrow B_{p, \infty}^{k}\left(\mathbb{R}^{n}, E\right) \quad(1 \leq p \leq \infty),
$$

and some properties of Banach space interpolation, we will show that the $W_{p}^{k}$-realization of parabolic pseudodifferential operators are the negative generators of analytic semigroups on $W_{p}^{k}\left(\mathbb{R}^{n}, E_{0}\right)$ with $1 \leq p \leq \infty$ (see Theorem 3.14). Therefore, we obtain the existence and uniqueness of solutions for a non-autonomous Cauchy problem in Sobolev spaces $W_{p}^{k}\left(\mathbb{R}^{n}, E\right)$ (see Theorem 4.3).

For vector-valued differential operators, generation of an analytic semigroup on $L_{p}\left(\mathbb{R}^{n}, E_{0}\right), C_{0}\left(\mathbb{R}^{n}, E_{0}\right)$ and $B U C\left(\mathbb{R}^{n}, E_{0}\right)$ was shown by Amann in [Am01]. Pseudodifferential operators with smooth symbols (with respect to the dual variable $\xi$ ) were considered in [Ki03]. In [Ki01], the generation of an analytic semigroup in $L_{p}\left(\mathbb{R}^{n}, E_{0}\right)(1 \leq p<\infty)$ was shown for suitable parabolic vector-valued pseudodifferential operators where the symbols are assumed to have a homogeneous principal part and regularity greater than or equal to $2 n+1$. Apart from generalizing these results, the method used in the present paper will allow us to consider $x$-dependent symbols in a forthcoming publication.

The plan of the paper is as follows: After some preliminary definitions and remarks in Section 2, we prove in Section 3 the main results of the present paper on continuity (Theorem 3.1) and on generation of an analytic semigroup (Theorem 3.14). As an application, we prove in Theorem 4.3 the existence and uniqueness of the solution of a non-autonomous Cauchy problem in the Sobolev spaces $W_{p}^{k}\left(\mathbb{R}^{n}, E\right)$. The constants $C_{1}, \ldots, C_{15}, M_{1}$ are rigourously calculated, because we will explore stability conditions for an adequate family of pseudodifferential operators in Remark 4.4.

\section{Preliminary Definitions AND REMARKS}

In the following, $E$ and $E_{i}$ always denote arbitrary Banach spaces with norm $\|\cdot\|_{E}$ and $\|\cdot\|_{E_{i}}$, respectively, and $\mathcal{L}\left(E_{1}, E_{0}\right)$ the space of linear, continuous maps of $E_{1}$ into $E_{0}$. The definitions and properties of functions spaces are taken from [Am97]. In particular, $\mathcal{S}\left(\mathbb{R}^{n}, E\right)$ denotes the Schwartz space of rapidly decrasing functions, $C_{b}^{k}\left(\mathbb{R}^{n}, E\right), k \in \mathbb{N}_{0}$, is the space of all functions $u: \mathbb{R}^{n} \rightarrow E$ such that $\partial^{\alpha} u$ is bounded and continuous on $\mathbb{R}^{n}$ for all $|\alpha| \leq k, B U C^{k}\left(\mathbb{R}^{n}, E\right)$ is the space of all $u \in C^{k}\left(\mathbb{R}^{n}, E\right)$ such that $\partial^{\alpha} u$ is bounded and uniformly continuous on $\mathbb{R}^{n}$ for all $|\alpha| \leq k$ and $B U C\left(\mathbb{R}^{n}, E\right):=B U C^{0}\left(\mathbb{R}^{n}, E\right)$.

The Besov space $B_{p, q}^{s}$, the homogeneous Besov space $\stackrel{\circ}{p}_{p, q}^{s}$ and the small Besov space spaces $b_{p, q}^{s}$ are defined as follows: For $s \in \mathbb{R}$ and $p, q \in[1, \infty]$ one defines the $E$-valued Besov space of order $s$ by

$$
B_{p, q}^{s}\left(\mathbb{R}^{n}, E\right):=\left\{u \in \mathcal{S}^{\prime}\left(\mathbb{R}^{n}, E\right):\left\|2^{j s}\right\| \psi_{j}(D) u\left\|_{L_{p}\left(\mathbb{R}^{n}, E\right)}\right\|_{l^{q}}<\infty\right\}
$$


with norm

$$
\|u\|_{B_{p, q}^{s}\left(\mathbb{R}^{n}, E\right)}:=\left\|2^{j s}\right\| \psi_{j}(D) u\left\|_{L_{p}\left(\mathbb{R}^{n}, E\right)}\right\|_{l^{q}},
$$

where $\mathcal{S}^{\prime}\left(\mathbb{R}^{n}, E\right)$ is the space of the $E$-valued tempered distributions,

$$
\psi_{j}(D) u:=\mathcal{F}^{-1}\left(\psi_{j} \mathcal{F} u\right)
$$

and $\left(\psi_{j}\right)_{j \in \mathbb{N}_{0}}$ is a resolution of unity which is constructed in the following way: For $\psi \in \mathcal{S}\left(\mathbb{R}^{n}\right):=\mathcal{S}\left(\mathbb{R}^{n}, \mathbb{C}\right)$ with

$$
\operatorname{supp} \psi \subset\left\{x \in \mathbb{R}^{n}:|x| \leq 2\right\} \text { and } \psi(x)=1 \text { on }|x| \leq 1,
$$

one defines $\widetilde{\psi}(x):=\psi(x)-\psi(2 x), \psi_{j}(x):=\widetilde{\psi}\left(2^{-j} x\right)$ for $x \in \mathbb{R}^{n}$ and $\psi_{0}:=\psi$, $\psi_{-1}:=0$. The sequence $\left(\psi_{j}\right)_{j \in \mathbb{N}_{0}}$ satisfies:

$$
\begin{aligned}
& \operatorname{supp}\left(\psi_{0}\right) \subset \Omega_{0}:=\left\{x \in \mathbb{R}^{n}:|x| \leq 2\right\}, \\
& \operatorname{supp}\left(\psi_{j}\right) \subset \Omega_{j}:=\left\{x \in \mathbb{R}^{n}: 2^{j-1} \leq|x| \leq 2^{j+1}\right\} \quad \text { for all } j \in \mathbb{N},
\end{aligned}
$$

as well as

$$
\sum_{j=0}^{\infty} \psi_{j}(\xi)=1 \text { for all } \xi \in \mathbb{R}^{n} .
$$

Moreover, for each $\alpha \in \mathbb{N}_{0}^{n}$ there exists a constant $c_{\alpha}>0$ such that ${ }^{1}$

$$
\left|D_{\xi}^{\alpha} \psi_{j}(\xi)\right| \leq c_{\alpha} 2^{-j|\alpha|} 1_{\Omega_{j}}(\xi) \text { for all } \xi \in \mathbb{R}^{n} \text { and } j \in \mathbb{N}_{0} .
$$

One defines the Banach spaces $\stackrel{\circ}{p, q}_{p, q}\left(\mathbb{R}^{n}, E\right)$ and $b_{p, q}^{s}\left(\mathbb{R}^{n}, E\right)$ as the closures of $\mathcal{S}\left(\mathbb{R}^{n}, E\right)$ and $B_{p, q}^{s+1}\left(\mathbb{R}^{n}, E\right)$, respectively, where the closure is taken with respect to the norm $\|\cdot\|_{B_{p, q}^{s}}$.

The following three lemmas will be crucial for the main results in Section 3.

Lemma 2.1. Let $s \in \mathbb{R}$ and $p, q \in[1, \infty]$. Then we have:

a) $\mathcal{S}\left(\mathbb{R}^{n}, E\right) \hookrightarrow B_{p, q}^{s}\left(\mathbb{R}^{n}, E\right) \hookrightarrow \mathcal{S}^{\prime}\left(\mathbb{R}^{n}, E\right)$ and $\mathcal{S}\left(\mathbb{R}^{n}, E\right) \stackrel{d}{\hookrightarrow} B_{p, q}^{s}\left(\mathbb{R}^{n}, E\right)$ if $p, q \in[1, \infty)$. Here $\hookrightarrow$ denotes continuous embedding and $\stackrel{d}{\hookrightarrow}$ denotes continuous and dense embedding.

b) For each $1 \leq q_{0} \leq q_{1} \leq \infty$,

$$
B_{p, q_{0}}^{s}\left(\mathbb{R}^{n}, E\right) \hookrightarrow B_{p, q_{1}}^{s}\left(\mathbb{R}^{n}, E\right) .
$$

c) For each $\varepsilon>0$ and $1 \leq q_{0}, q_{1} \leq \infty$,

$$
B_{p, q_{0}}^{s+\varepsilon}\left(\mathbb{R}^{n}, E\right) \hookrightarrow B_{p, q_{1}}^{s}\left(\mathbb{R}^{n}, E\right) .
$$

d) Let $s_{1} \leq s_{0}$ and $1 \leq p_{0} \leq p_{1} \leq \infty$ with $s_{1}-\frac{n}{p_{1}}=s_{0}-\frac{n}{p_{0}}$. Then

$$
B_{p_{0}, q}^{s_{0}}\left(\mathbb{R}^{n}, E\right) \hookrightarrow B_{p_{1}, q}^{s_{1}}\left(\mathbb{R}^{n}, E\right) .
$$

e)

$$
b_{p, q}^{s}\left(\mathbb{R}^{n}, E\right)= \begin{cases}B_{p, q}^{s}\left(\mathbb{R}^{n}, E\right), & \text { if } 1 \leq p \leq \infty, 1 \leq q<\infty, \\ B_{p, \infty}^{s}\left(\mathbb{R}^{n}, E\right), & \text { if } 1 \leq p<\infty, q=\infty .\end{cases}
$$

f) For $1 \leq p<\infty$ and $k \in \mathbb{N}$,

$$
\stackrel{\circ}{B}_{p, 1}^{k}\left(\mathbb{R}^{n}, E\right) \stackrel{d}{\hookrightarrow} W_{p}^{k}\left(\mathbb{R}^{n}, E\right) \stackrel{d}{\hookrightarrow} \stackrel{\circ}{B}_{p, \infty}^{k}\left(\mathbb{R}^{n}, E\right)=b_{p, \infty}^{k}\left(\mathbb{R}^{n}, E\right),
$$

\footnotetext{
${ }^{1} 1_{\Omega}$ denotes the characteristic function of a set $\Omega$.
} 
6 PSEUDODIFFERENTIAL OPERATORS ON VECTOR-VALUED SOBOLEV SPACES

$$
\begin{gathered}
B_{\infty, 1}^{k}\left(\mathbb{R}^{n}, E\right) \hookrightarrow W_{\infty}^{k}\left(\mathbb{R}^{n}, E\right) \hookrightarrow B_{\infty, \infty}^{k}\left(\mathbb{R}^{n}, E\right), \\
b_{\infty, 1}^{k}\left(\mathbb{R}^{n}, E\right)=B_{\infty, 1}^{k}\left(\mathbb{R}^{n}, E\right) \stackrel{d}{\hookrightarrow} B U C^{k}\left(\mathbb{R}^{n}, E\right) \stackrel{d}{\hookrightarrow} b_{\infty, \infty}^{k}\left(\mathbb{R}^{n}, E\right), \\
\stackrel{\circ}{\infty, 1}_{\infty}\left(\mathbb{R}^{n}, E\right) \stackrel{d}{\hookrightarrow} C_{0}^{k}\left(\mathbb{R}^{n}, E\right) \stackrel{d}{\hookrightarrow} \stackrel{\circ}{\infty, \infty}_{\infty}^{k}\left(\mathbb{R}^{n}, E\right), \\
B_{\infty, 1}^{k}\left(\mathbb{R}^{n}, E\right) \hookrightarrow C_{b}^{k}\left(\mathbb{R}^{n}, E\right) \hookrightarrow B_{\infty, \infty}^{k}\left(\mathbb{R}^{n}, E\right) .
\end{gathered}
$$

g) If $p, q, q_{1}, q_{2} \in[1, \infty],-\infty<s_{1}<s_{2}<\infty$ and $\theta \in(0,1)$, then ${ }^{2}$

$$
\left(B_{p, q_{1}}^{s_{1}}\left(\mathbb{R}^{n}, E\right), B_{p, q_{2}}^{s_{2}}\left(\mathbb{R}^{n}, E\right)\right)_{\theta, q} \cong B_{p, q}^{(1-\theta) s_{1}+\theta s_{2}}\left(\mathbb{R}^{n}, E\right) .
$$

Proof. See [Am95], Chap. 5 or [Sc86].

By Definition and Lemma 2.1 a), it follows immediately that

$$
\begin{aligned}
\mathcal{S}\left(\mathbb{R}^{n}, E\right) \stackrel{d}{\hookrightarrow} \stackrel{\circ}{B}_{p, q}^{s}\left(\mathbb{R}^{n}, E\right) \hookrightarrow B_{p, q}^{s}\left(\mathbb{R}^{n}, E\right) & (s \in \mathbb{R}, p, q \in[1, \infty]), \\
B_{p, q}^{s+1}\left(\mathbb{R}^{n}, E\right) \stackrel{d}{\hookrightarrow} b_{p, q}^{s}\left(\mathbb{R}^{n}, E\right) \hookrightarrow B_{p, q}^{s}\left(\mathbb{R}^{n}, E\right) & (s \in \mathbb{R}, p, q \in[1, \infty]), \\
\dot{B}_{p, q}^{s}\left(\mathbb{R}^{n}, E\right)=B_{p, q}^{s}\left(\mathbb{R}^{n}, E\right) & (s \in \mathbb{R}, p, q \in[1, \infty)) .
\end{aligned}
$$

Also one can easily see, using again the previous Lemma, that if $\mathcal{B} \in$ $\{b, \stackrel{\circ}{B}, B\}, 1 \leq p, q_{1}, q_{2} \leq \infty, t>s$ and $E_{1} \hookrightarrow E_{0}$, then

$$
\mathcal{B}_{p, q_{1}}^{t}\left(\mathbb{R}^{n}, E_{1}\right) \hookrightarrow \mathcal{B}_{p, q_{2}}^{s}\left(\mathbb{R}^{n}, E_{0}\right) .
$$

If $E_{1} \stackrel{d}{\hookrightarrow} E_{0}$, the continuous embedding (20) is always dense, except when $\mathcal{B}=B$ and $q_{1}$ or $q_{2}$ is $\infty$. Moreover

$$
\mathcal{B}_{p, q_{1}}^{s}\left(\mathbb{R}^{n}, E_{1}\right) \hookrightarrow \mathcal{B}_{p, q_{2}}^{s}\left(\mathbb{R}^{n}, E_{0}\right) \quad\left(1 \leq q_{1} \leq q_{2} \leq \infty\right) .
$$

Lemma 2.2. Let $s \in \mathbb{R}, p \in[1, \infty]$ and $q \in[1, \infty)$. Then

$$
\left(C_{b}^{\infty}\left(\mathbb{R}^{n}, E\right) \cap B_{p, q}^{s}\left(\mathbb{R}^{n}, E\right) ;\|\cdot\|_{B_{p, q}^{s}}\right) \stackrel{d}{\hookrightarrow} B_{p, q}^{s}\left(\mathbb{R}^{n}, E\right) .
$$

Proof. For all $s \in \mathbb{R}, p \in[1, \infty]$ and $q \in[1, \infty)$ it is clear that

$$
\left(C_{b}^{\infty}\left(\mathbb{R}^{n}, E\right) \cap B_{p, q}^{s}\left(\mathbb{R}^{n}, E\right) ;\|\cdot\|_{B_{p, q}^{s}}\right) \hookrightarrow B_{p, q}^{s}\left(\mathbb{R}^{n}, E\right) .
$$

It only remains to prove the density. In the case $p<\infty$, the density in (22) follows from Lemma 2.1 a) and $\mathcal{S}\left(\mathbb{R}^{n}, E\right) \subset C_{b}^{\infty}\left(\mathbb{R}^{n}, E\right) \cap B_{p, q}^{s}\left(\mathbb{R}^{n}, E\right)$. Now, let $u \in B_{\infty, q}^{s}\left(\mathbb{R}^{n}, E\right)$ with $q \in[1, \infty),\left(\psi_{j}\right)_{j \in \mathbb{N}_{0}}$ a partition of unity as in (8) and $u_{N}:=\sum_{k=0}^{N} \psi_{k}(D) u, N \in \mathbb{N}$. By definition $(4), \psi_{j}(D) u \in L_{\infty}\left(\mathbb{R}^{n}, E\right)$ for all $j \in \mathbb{N}_{0}$, and therefore $u_{N} \in L_{\infty}\left(\mathbb{R}^{n}, E\right)$. On the other hand, let $\left(\varphi_{j}\right)_{j \in \mathbb{N}_{0}}$ be another partition of the unity as in (8). Then one obtains from (5) and the fact that convolution corresponds to multiplication of the Fourier transform ${ }^{3}$ that

$$
\varphi_{j}(D)\left(\psi_{k}(D) u\right)=(2 \pi)^{-\frac{n}{2}} \check{\varphi}_{j} *\left(\psi_{k}(D) u\right) \in \mathcal{O}_{\mathcal{M}}\left(\mathbb{R}^{n}, E\right)
$$

\footnotetext{
${ }^{2}$ Here $\left(E_{0}, E_{1}\right)_{\theta, q}$ denotes the real interpolation space with exponent $\theta$ and parameter $q$ between the Banach spaces $E_{1}$ and $E_{0}$.

${ }^{3} \mathcal{O}_{\mathcal{M}}\left(\mathbb{R}^{n}, E\right)$ denotes the space of $E$-valued slowly increasing smooth functions on $\mathbb{R}^{n}$. For the result on convolution see [Am97], Th. 3.6.
} 
for all $j, k \in \mathbb{N}_{0}$ where $\check{\varphi}_{j}:=\mathcal{F}^{-1} \varphi_{j}$. Therefore $\varphi_{j}(D)\left(\psi_{k}(D) u\right)$ is a regular tempered distribution. From $(23), \check{\varphi}_{j} \in L_{1}\left(\mathbb{R}^{n}\right), \psi_{k}(D) u \in L_{\infty}\left(\mathbb{R}^{n}, E_{0}\right)$ and Theorem 1.9.9 in [Am03] it follows that for all $j, k \in \mathbb{N}_{0}$

$$
\left\|\varphi_{j}(D)\left(\psi_{k}(D) u\right)\right\|_{L_{\infty}} \leq\left\|\check{\varphi}_{j}\right\|_{L_{1}}\left\|\psi_{k}(D) u\right\|_{L_{\infty}}=c_{\varphi}\left\|\psi_{k}(D) u\right\|_{L_{\infty}}
$$

where the constant $c_{\varphi}:=\max \left\{\|\check{\varphi}\|_{L_{1}},\left\|(\widetilde{\varphi})^{\vee}\right\|_{L_{1}}\right\}$ is independent of $j$ and $k$. Thus

$$
\varphi_{j}(D)\left(u-u_{N}\right)=\varphi_{j}(D) u-\sum_{k=0}^{N} \varphi_{j}(D)\left(\psi_{k}(D) u\right) \in L_{\infty}\left(\mathbb{R}^{n}, E\right) .
$$

From $\operatorname{supp}\left(\psi_{j}\right), \operatorname{supp}\left(\varphi_{j}\right) \subset \Omega_{j},(24)$ and $\sum_{k=0}^{\infty} \psi_{k}(D) u=u$ in $\mathcal{S}^{\prime}\left(\mathbb{R}^{n}, E\right)$, we see that

$$
\begin{aligned}
\| u- & u_{N} \|_{B_{\infty, q}^{s}\left(\mathbb{R}^{n}, E\right)}^{q} \\
= & \sum_{j=0}^{\infty} 2^{j s q}\left\|\varphi_{j}(D)\left(u-\sum_{k=0}^{N} \mathcal{F}^{-1}\left(\psi_{k} \mathcal{F} u\right)\right)\right\|_{L_{\infty}\left(\mathbb{R}^{n}, E\right)}^{q} \\
= & \sum_{j=0}^{\infty} 2^{j s q}\left\|\varphi_{j}(D)\left(\sum_{k=0}^{\infty} \mathcal{F}^{-1}\left(\psi_{k} \mathcal{F} u\right)-\sum_{k=0}^{N} \mathcal{F}^{-1}\left(\psi_{k} \mathcal{F} u\right)\right)\right\|_{L_{\infty}\left(\mathbb{R}^{n}, E\right)}^{q} \\
& \stackrel{(5)}{=} \sum_{j=0}^{\infty} 2^{j s q}\left\|\mathcal{F}^{-1}\left(\varphi_{j} \sum_{k=N+1}^{\infty} \psi_{k} \mathcal{F} u\right)\right\|_{L_{\infty}\left(\mathbb{R}^{n}, E\right)}^{q} \\
= & 2^{N s q}\left\|\mathcal{F}^{-1}\left(\varphi_{N} \psi_{N+1} \mathcal{F} u\right)\right\|_{L_{\infty}\left(\mathbb{R}^{n}, E\right)}^{q} \\
& +2^{(N+1) s q}\left\|\mathcal{F}^{-1}\left(\varphi_{N+1}\left(\psi_{N+1}+\psi_{N+2}\right) \mathcal{F} u\right)\right\|_{L_{\infty}\left(\mathbb{R}^{n}, E\right)}^{q} \\
& \left.+\sum_{j=N+2}^{\infty} 2^{j s q} \| \mathcal{F}^{-1}\left(\varphi_{j} \mathcal{F} u\right)\right] \|_{L_{\infty}\left(\mathbb{R}^{n}, E\right)}^{q}\left(\text { since } \sum_{r=-1}^{1} \varphi_{j} \psi_{j+r}=\varphi_{j}\right) \\
& \stackrel{(5)}{=} 2^{N s q}\left\|\psi_{N+1}(D)\left(\varphi_{N}(D) u\right)\right\|_{L_{\infty}}^{q} \\
& +2^{(N+1) s q}\left\|\left(\psi_{N+1}+\psi_{N+2}\right)(D)\left(\varphi_{N+1}(D) u\right)\right\|_{\infty}^{q} \\
& \left.+\sum_{j=N+2}^{\infty} 2^{j s q}\left\|\varphi_{j}(D) u\right\|_{\infty}^{q}\right\} \\
& (24) \\
\leq & c_{n, q, s, \psi}\left\{2^{N s q}\left\|\varphi_{N}(D) u\right\|_{\infty}^{q}+2^{(N+1) s q}\left\|\left(\varphi_{N+1}(D) u\right)\right\|_{\infty}^{q}\right. \\
& \left.+\sum_{j=N+q, s, \psi}^{\infty} \sum_{j=N}^{\infty} 2^{j s q}\left\|\varphi_{j}(D) u\right\|_{\infty}^{q}\right\} \\
& \left.{ }_{j}\right\} \varphi_{j}(D) u \|_{\infty}^{q} \underset{N \rightarrow \infty}{\longrightarrow} 0,
\end{aligned}
$$

because $u$ is in $B_{\infty, q}^{s}\left(\mathbb{R}^{n}, E\right)$. This implies that $u_{N} \in B_{\infty, q}^{s}\left(\mathbb{R}^{n}, E\right)$ and $u_{N} \rightarrow$ $u$ in $B_{\infty, q}^{s}\left(\mathbb{R}^{n}, E\right)$. Furthermore $u_{N}$ is a regular distribution in $C_{b}^{\infty}\left(\mathbb{R}^{n}, E\right)$, 
because we have, setting $\chi_{k}:=\sum_{r=-1}^{1} \psi_{k+r}$,

$$
\begin{aligned}
u_{N} & =\sum_{k=0}^{N} \mathcal{F}^{-1}\left(\psi_{k} \mathcal{F} u\right)=\sum_{k=0}^{N} \mathcal{F}^{-1}\left(\left(\chi_{k} \psi_{k}\right) \mathcal{F} u\right) \\
& =\sum_{k=0}^{N} \mathcal{F}^{-1}\left(\chi_{k} \mathcal{F}\left(\psi_{k}(D) u\right)\right)=\sum_{k=0}^{N} c_{n} \check{\chi}_{k} *\left(\psi_{k}(D) u\right) .
\end{aligned}
$$

Thus, it follows from Theorem 1.9.9 in [Am03], for each $\alpha \in \mathbb{N}_{0}^{n}$, that

$$
\partial^{\alpha} u_{N}=c_{n} \sum_{k=0}^{N} \underbrace{\left(\partial^{\alpha} \check{\chi}_{k}\right)}_{\in L_{1}} * \underbrace{\left(\psi_{k}(D) u\right)}_{\in L_{\infty}} \in B U C\left(\mathbb{R}^{n}, E\right) .
$$

This finishes the proof.

Lemma 2.3. Let $\left(\psi_{j}\right)_{j \in \mathbb{N}_{0}}$ as in (8), $B:=\left\{x \in \mathbb{R}^{n}: \frac{1}{2} \leq|x| \leq 2\right\}, m \in \mathbb{R}$ and $a \in S_{1,0}^{m, n+1}\left(\mathbb{R}^{n}, E\right)$. Then $\left(\psi_{j} a\right)^{\vee} \in L_{1}\left(\mathbb{R}^{n}, E\right)$ and

$$
\left\|\left(\psi_{j} a\right)^{\vee}\right\|_{L_{1}\left(\mathbb{R}^{n}, E\right)} \leq C_{1} 2^{j m}\|a\|_{S_{1,0}^{m, n+1}\left(\mathbb{R}^{n}, E\right)} \quad\left(j \in \mathbb{N}_{0}\right),
$$

where the constant $C_{1}$ is given by $C_{1}:=2^{\frac{3 m}{2}} \omega_{n} C_{1}^{*} \max \left\{\operatorname{vol}(B), \sum_{|\alpha|=n+1} n^{\frac{n+1}{2}}\right\}$ with $C_{1}^{*}:=\|\widetilde{\psi}\|_{C_{b}^{n+1}} \sum_{\substack{|\alpha|=n+1 \\ \beta \leq \alpha}}\left(\begin{array}{l}\alpha \\ \beta\end{array}\right) 2^{|\beta|}$ and $\omega_{n}$ denoting the volume of the unit ball in $\mathbb{R}^{n}$.

Proof. For all $j \in \mathbb{N}_{0}$, we have

$$
\left\|\left(\psi_{j} a\right)^{\vee}\right\|_{L_{1}\left(\mathbb{R}^{n}, E\right)}=\left\|\left(\widetilde{\psi} a\left(2^{j} \cdot\right)\right)^{\vee}\right\|_{L_{1}\left(\mathbb{R}^{n}, E\right)}
$$

where $\widetilde{\psi}=\psi$ in the case $j=0$. Now, let $1_{B}$ be the characteristic function of $B$, and let $\alpha \in \mathbb{N}_{0}^{n}$ with $|\alpha| \leq n+1$ and $j \in \mathbb{N}$. Then for all $\xi \in \mathbb{R}^{n}$ we obtain $^{4}$

$$
\begin{aligned}
& \left\|D^{\alpha}\left(\widetilde{\psi} a\left(2^{j} \cdot\right)\right)(\xi)\right\|_{E} \\
& \quad \leq \sum_{\beta \leq \alpha}\left(\begin{array}{c}
\alpha \\
\beta
\end{array}\right) \underbrace{2^{j|\beta|}}_{=2^{|\beta| 2^{(j-1)|\beta|}}}\left|D^{\alpha-\beta} \widetilde{\psi}(\xi)\right| 1_{B}(\xi)\left\|\left(D^{\beta} a\right)\left(2^{j} \xi\right)\right\|_{E} \\
& \quad \leq \sum_{\beta \leq \alpha}\left(\begin{array}{l}
\alpha \\
\beta
\end{array}\right) 2^{|\beta|}\|\widetilde{\psi}\|_{C_{b}^{n+1}} 1_{B}(\xi) \max _{|\beta| \leq n+1} \sup _{\Omega_{j}}\langle\xi\rangle^{|\beta|}\left\|D^{\beta} a(\xi)\right\|_{E} \\
& \quad \leq C_{1}^{*}\|a\|_{S_{1,0}^{m, n+1}} 1_{B}(\xi) \sup _{\Omega_{j}}\langle\xi\rangle^{m} \\
& \quad \leq C_{1}^{*}\|a\|_{S_{1,0}^{m, n+1}} 1_{B}(\xi) \cdot\left\{\begin{array}{l}
\left(1+2^{2(j+1)}\right)^{m / 2} \text { if } m \geq 0, \\
\left(1+2^{2(j-1)}\right)^{m / 2} \text { if } m<0, \\
\quad \leq 2^{\frac{3 m}{2}} C_{1}^{*} 2^{j m}\|a\|_{S_{1,0}^{m, n+1}} 1_{B}(\xi) .
\end{array}\right.
\end{aligned}
$$

An analogous result is obtained for $j=0$ with $\widetilde{\psi}=\psi$. Therefore,

$$
D^{\alpha}\left(\widetilde{\psi} a\left(2^{j} \cdot\right)\right) \in L_{1}\left(\mathbb{R}^{n}, E\right),
$$

\footnotetext{
${ }^{4} D^{\alpha}:=(-i)^{|\alpha|} \partial^{\alpha}$.
} 

and for all $j \in \mathbb{N}_{0}$ and $|\alpha| \leq n+1$ we have

$$
\left\|D^{\alpha}\left(\widetilde{\psi} a\left(2^{j} \cdot\right)\right)\right\|_{L_{1}\left(\mathbb{R}^{n}, E\right)} \leq 2^{\frac{3 m}{2}} C_{1}^{*} 2^{j m}\|a\|_{S_{1,0}^{m, n+1}} .
$$

From the last inequality, the equality

$$
x^{\alpha} \mathcal{F}^{-1}\left(\widetilde{\psi} a\left(2^{j} \cdot\right)\right)=(-1)^{|\alpha|} \mathcal{F}^{-1}\left(D^{\alpha}\left(\widetilde{\psi} a\left(2^{j} \cdot\right)\right)\right) \in C_{0}\left(\mathbb{R}^{n}, E\right),
$$

(25) and the inequality $|x|^{n+1} \leq n^{\frac{n+1}{2}} \sum_{|\alpha|=n+1}\left|x^{\alpha}\right|$ one concludes that

$$
\begin{aligned}
& \left\|\left(\psi_{j} a\right)^{\vee}\right\|_{L_{1}\left(\mathbb{R}^{n}, E\right)} \\
& =\int_{B_{1}(0)}\left\|\mathcal{F}^{-1}\left(\widetilde{\psi} a\left(2^{j} \cdot\right)\right)(x)\right\|_{E} d x+\int_{\mathbb{R}^{n} \backslash B_{1}(0)}\left\|\mathcal{F}^{-1}\left(\widetilde{\psi} a\left(2^{j} \cdot\right)\right)(x)\right\|_{E} d x \\
& \leq \int_{B_{1}(0)} \int_{B}\left(|\widetilde{\psi}(\xi)|\left\|a\left(2^{j} \xi\right)\right\|_{E} d \xi\right) d x \\
& \quad+\int_{\mathbb{R}^{n} \backslash B_{1}(0)} \frac{n^{\frac{n+1}{2}} \sum_{|\alpha|=n+1}\left|x^{\alpha}\right|}{|x|^{n+1}}\left\|\mathcal{F}^{-1}\left(\widetilde{\psi} a\left(2^{j} \cdot\right)\right)(x)\right\|_{E} d x \\
& \leq 2^{\frac{3 m}{2}} C_{1}^{*} \omega_{n} \operatorname{vol}(B) 2^{j m}\|a\|_{S_{1,0}^{m, n+1}} \\
& \quad+\sum_{|\alpha|=n+1} n^{\frac{n+1}{2}} \int_{\mathbb{R}^{n} \backslash B_{1}(0)} \frac{1}{|x|^{n+1}}\left\|\mathcal{F}^{-1}\left(D^{\alpha}\left(\widetilde{\psi} a\left(2^{j} \cdot\right)\right)\right)(x)\right\|_{E} d x \\
& \leq 2^{\frac{3 m}{2}} C_{1}^{*} \omega_{n} \operatorname{vol}(B) 2^{j m}\|a\|_{S_{1,0}^{m, n+1}} \\
& \quad+\sum_{|\alpha|=n+1} n^{\frac{n+1}{2}} \int_{\mathbb{R}^{n} \backslash B_{1}(0)} \frac{1}{|x|^{n+1}}\left(\int_{B}\left\|D^{\alpha}\left(\widetilde{\psi} a\left(2^{j} \cdot\right)\right)(\xi)\right\|_{E} d \xi\right) d x \\
& \leq 2^{\frac{3 m}{2}} C_{1}^{*} \omega_{n} \operatorname{vol}(B) 2^{j m}\|a\|_{S_{1,0}^{m, n+1}} \\
& \quad+2^{\frac{3 m}{2}} C_{1}^{*}\left(\sum_{|\alpha|=n+1} n^{\frac{n+1}{2}}\right) 2^{j m}\|a\|_{S_{1,0}^{m, n+1}} \int_{\mathbb{R}^{n} \backslash B_{1}(0)} \frac{1}{|x|^{n+1}} d x \\
& \leq C_{1} 2^{j m}\|a\|_{S_{1,0}^{m, n+1}},
\end{aligned}
$$

with $C_{1}:=2^{\frac{3 m}{2}} \omega_{n} C_{1}^{*} \max \left\{\operatorname{vol}(B), \sum_{|\alpha|=n+1} n^{\frac{n+3}{2}}\right\}$.

Remark 2.4. Let $a \in S_{1,0}^{m, \rho}\left(\mathbb{R}^{n}, E_{1}\right)$ and $u \in C_{b}^{\infty}\left(\mathbb{R}^{n}, E_{2}\right)$. For the definition of

$$
[a(D) u](x)=\mathrm{Os}-\iint_{\mathbb{R}^{2 n}} e^{i \xi \cdot \eta} a(\xi) \bullet u(x-\eta) \frac{d(\xi, \eta)}{(2 n)^{n}}, \quad x \in \mathbb{R}^{n},
$$

(cf. [Ku81]), the condition $\rho \leq n+1$ is not sufficient, because

$$
b_{x}(\xi, \eta):=a(\xi) \bullet u(x-\eta) \in \mathcal{A}_{\delta, \tau}^{m}
$$

with $\delta=0$ and $\tau=0$, and the oscillatory integral in (26) exists if $n+\tau<2 l$ and $\frac{n+m}{1-\delta}<2 l^{\prime}$, where $2 l \quad\left(l \in \mathbb{N}_{0}\right)$ denotes the necessary number of derivatives in the variable $\xi$ (see $[\mathrm{Ku} 81]$ ). We obtain the condition $n<2 l$ 
and therefore we consider in this paper $\rho \geq \rho_{n}$, where $\rho_{n}$ is the smallest even number greater than $n$ (see (3)).

\section{MAin RESUltS}

Theorem 3.1 (and definition of the operator $\widetilde{a(D)}$ ). Let $m \in \mathbb{R}, a \in$ $S_{1,0}^{m, \rho_{n}}\left(\mathbb{R}^{n} ; E_{1}\right)$ and $\bullet: E_{1} \times E_{2} \rightarrow E_{0}$ a multiplication ${ }^{5}$. Then it holds:

a) There exists a map $T: \mathbf{V}\left(\mathbb{R}^{n}, E_{2}\right) \longrightarrow \mathbf{V}\left(\mathbb{R}^{n}, E_{0}\right)$, such that

i) $T$ is linear.

ii) $\left.T\right|_{B_{p, q}^{s+m}}: B_{p, q}^{s+m}\left(\mathbb{R}^{n}, E_{2}\right) \longrightarrow B_{p, q}^{s}\left(\mathbb{R}^{n}, E_{0}\right)$ is continuous for all $s \in \mathbb{R}$ and $p, q \in[1, \infty]$.

iii) $\left.T\right|_{\left(B_{p, q}^{s+m}\left(\mathbb{R}^{n}, E_{2}\right) \cap C_{b}^{\infty}\left(\mathbb{R}^{n}, E_{2}\right)\right)}=a(D)$ for all $(s, p, q) \in \mathbb{R} \times[1, \infty] \times$ $[1, \infty]$, where

$[a(D) u](x)=o s-\iint e^{i \xi \cdot \eta} a(\xi) \bullet u(x-\eta) \frac{d(\xi, \eta)}{(2 \pi)^{n}}, u \in C_{b}^{\infty}\left(\mathbb{R}^{n}, E_{2}\right)$.

b) Let $S: \mathbf{V}\left(\mathbb{R}^{n}, E_{2}\right) \longrightarrow \mathbf{V}\left(\mathbb{R}^{n}, E_{0}\right)$ be another map satisfaying $\left.i\right)$-iii). Then $T=S$. We denote this operator $T$ by $\widetilde{a(D)}$.

Proof. The proof of a) is done in four steps:

I) We will show that for all $(s,(p, q)) \in \mathbb{R} \times[1, \infty]^{2}$

$$
a(D):\left(B_{p, q}^{s+m}\left(\mathbb{R}^{n}, E_{2}\right) \cap C_{b}^{\infty}\left(\mathbb{R}^{n}, E_{2}\right) ;\|\cdot\|_{B_{p, q}^{s+m}\left(\mathbb{R}^{n}, E_{2}\right)}\right) \rightarrow B_{p, q}^{s}\left(\mathbb{R}^{n}, E_{0}\right)
$$

is continuous. For this purpose one calculates $\psi_{j}(D)(a(D) u)$ with $u \in B_{p, q}^{s+m}\left(\mathbb{R}^{n}, E_{2}\right) \cap C_{b}^{\infty}\left(\mathbb{R}^{n}, E_{2}\right):$

$$
\begin{aligned}
{\left[\psi_{j}(D)(a(D) u)\right](x) } & =\left[\left(\psi_{j} a\right)(D)\right] u(x) \\
& =(2 \pi)^{-\frac{n}{2}}[\underbrace{\left(\psi_{j} a\right)^{\vee} *_{\bullet} \chi_{j}(D) u}_{\in L_{p}\left(\mathbb{R}^{n}, E_{0}\right)}](x),
\end{aligned}
$$

where $\chi_{j}:=\sum_{i=-1}^{1} \psi_{j+i}$ and $*_{\bullet}$ is the convolution relative to the multiplication - (see [Am97]). From this and from Lemma 2.3 it follows for all $u \in B_{p, q}^{s+m}\left(\mathbb{R}^{n}, E_{2}\right) \cap C_{b}^{\infty}\left(\mathbb{R}^{n}, E_{2}\right)$ that

$$
\|a(D) u\|_{B_{p, q}^{s}\left(\mathbb{R}^{n}, E_{0}\right)} \leq C_{2}\|a\|_{S_{1,0}^{m, n+1}\left(\mathbb{R}^{n}, E_{1}\right)}\|u\|_{B_{p, q}^{s+m}\left(\mathbb{R}^{n}, E_{2}\right)},
$$

with $C_{2}:=3(2 \pi)^{-\frac{n}{2}} C_{1}$.

II) Because we have the embedding

$$
\left(B_{p, q}^{s}\left(\mathbb{R}^{n}, E\right) \cap C_{b}^{\infty}\left(\mathbb{R}^{n}, E\right) ;\|\cdot\|_{B_{p, q}^{s}}\right) \stackrel{d}{\hookrightarrow} B_{p, q}^{s}\left(\mathbb{R}^{n}, E\right)
$$

for all $1 \leq p \leq \infty$ and $1 \leq q<\infty$ (see Lemma 2.2), there exists a unique linear continuous extension

$$
a_{s, p, q}(D): B_{p, q}^{s+m}\left(\mathbb{R}^{n}, E_{2}\right) \rightarrow B_{p, q}^{s}\left(\mathbb{R}^{n}, E_{0}\right)
$$

of $a(D)$ giving I).

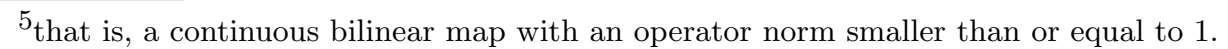


III) One defines $T: \mathbf{V}\left(\mathbb{R}^{n}, E_{2}\right) \longrightarrow \mathbf{V}\left(\mathbb{R}^{n}, E_{0}\right)$ by $\left.T\right|_{B_{p, q}^{s+m}}:=a_{s, p, q}(D)$ for all $(s, p, q) \in \mathbb{R} \times[1, \infty] \times[1, \infty)$ and by $a_{s-1, p, q}(D)$ in the other cases. $T$ is well-defined on $\mathbf{V}\left(\mathbb{R}^{n}, E_{2}\right)$ and fulfills $\left.a\right)$ for all $q<\infty$.

IV) The case $q=\infty$ can be treated using real interpolation theory. ${ }^{6}$ In fact, from $B_{p, \infty}^{s+m}\left(\mathbb{R}^{n}, E_{2}\right)=\left(B_{p, 1}^{s+m-1}\left(\mathbb{R}^{n}, E_{2}\right), B_{p, 1}^{s+m+1}\left(\mathbb{R}^{n}, E_{2}\right)\right)_{\frac{1}{2}, \infty}$ $B_{p, \infty}^{s}\left(\mathbb{R}^{n}, E_{0}\right)=\left(B_{p, 1}^{s-1}\left(\mathbb{R}^{n}, E_{0}\right), B_{p, 1}^{s+1}\left(\mathbb{R}^{n}, E_{0}\right)\right)_{\frac{1}{2}, \infty}$, and from

$$
\begin{array}{ccc}
B_{p, 1}^{s+m-1}\left(\mathbb{R}^{n}, E_{2}\right) & \stackrel{a_{s-1, p, 1}(D)}{\text { continuous }} & B_{p, 1}^{s-1}\left(\mathbb{R}^{n}, E_{0}\right) \\
\cup & \cup \\
B_{p, \infty}^{s+m}\left(\mathbb{R}^{n}, E_{2}\right) & \stackrel{a_{s-1, p, 1}(D)}{\longrightarrow} & B_{p, \infty}^{s}\left(\mathbb{R}^{n}, E_{0}\right) \\
\cup & & \cup \\
B_{p, 1}^{s+m+1}\left(\mathbb{R}^{n}, E_{2}\right) & \stackrel{a_{s-1, p, 1}(D)}{\text { continuous }} & B_{p, 1}^{s+1}\left(\mathbb{R}^{n}, E_{0}\right)
\end{array}
$$

it follows that $a_{s-1, p, 1}(D): B_{p, \infty}^{s+m}\left(\mathbb{R}^{n}, E_{2}\right) \rightarrow B_{p, \infty}^{s}\left(\mathbb{R}^{n}, E_{0}\right)$ is continouos. To prove $b)$, let $u \in \mathbf{V}\left(\mathbb{R}^{n}, E_{2}\right)$. Then there exists a $(s, p, q) \in$ $\mathbb{R} \times[1, \infty] \times[1, \infty)$ such that $u \in B_{p, q}^{s+m}\left(\mathbb{R}^{n}, E_{2}\right)$. From this, (27) and a) assertion b) follows.

Corollary 3.2. Let $m, s \in \mathbb{R}, 1 \leq p, q \leq \infty$ and $\mathcal{B} \in\{B, \stackrel{\circ}{B}, b\}$. Then

$$
(a \mapsto \widetilde{a(D)}) \in \mathcal{L}\left(S_{1,0}^{m, \rho_{n}}\left(\mathbb{R}^{n}, E_{1}\right), \mathcal{L}\left(\mathcal{B}_{p, q}^{m+s}\left(\mathbb{R}^{n}, E_{2}\right), \mathcal{B}_{p, q}^{s}\left(\mathbb{R}^{n}, E_{0}\right)\right)\right)
$$

with

$$
\|\widetilde{a(D)} u\|_{\mathcal{B}_{p, q}^{s}\left(\mathbb{R}^{n} ; E_{0}\right)} \leq C_{3}\|a\|_{S_{1,0}^{m, n+1}\left(\mathbb{R}^{n} ; E_{1}\right)}\|u\|_{\mathcal{B}_{p, q}^{s+m}\left(\mathbb{R}^{n} ; E_{2}\right)}
$$

for all $u \in \mathcal{B}_{p, q}^{s+m}\left(\mathbb{R}^{n} ; E_{2}\right)$, where $C_{3}=3(2 \pi)^{-\frac{n}{2}} C_{1}$.

3.1. Analytic semigroups. In this subsection we will assume that $E_{1} \hookrightarrow$ $E_{0}$. For $s \in \mathbb{R}, p, q \in[1, \infty], m \in \mathbb{R}^{+}, \mathcal{B} \in\{B, \stackrel{\circ}{B}, b\}$ and $a \in S_{1,0}^{m, \rho_{n}}\left(\mathbb{R}^{n}, \mathcal{L}\left(E_{1}, E_{0}\right)\right)$, we denote $A:=\left.\widetilde{a(D)}\right|_{\mathcal{B}_{p, q}^{s+m}\left(\mathbb{R}^{n}, E_{1}\right)}$ with domain $D(A)=\mathcal{B}_{p, q}^{s+m}\left(\mathbb{R}^{n}, E_{1}\right)$ and $E:=\mathcal{B}_{p, q}^{s}\left(\mathbb{R}^{n}, E_{0}\right)$. Since $E_{1} \hookrightarrow E_{0}$ and $m>0$, it holds that

$$
D(A) \hookrightarrow \mathcal{B}_{p, q}^{s+m}\left(\mathbb{R}^{n}, E_{0}\right) \hookrightarrow \mathcal{B}_{p, q}^{s}\left(\mathbb{R}^{n}, E_{0}\right)=E .
$$

From this and Corollary 3.2 it follows that

$$
A: D(A) \subset E \rightarrow E \text { is a continuous linear operator. }
$$

These notations will be used in Proposition 3.5 and Corollary 3.6 to prove that the operator $-A$ in (28) is sectorial and hence it generates an analytic semigroup on $\mathcal{B}_{p, q}^{s}\left(\mathbb{R}^{n}, E_{0}\right)$, when the symbol $a$ is parabolic in the sense of Definition 3.4. In the following we introduce the notation $\rho(A)$ for the resolvent set of $A, R(\lambda, A):=(\lambda I-A)^{-1}$, with $\lambda \in \rho(A)$, for the resolvent operator of $A$ and

$$
\mathcal{L}_{i s}\left(E_{1}, E_{0}\right):=\left\{T \in \mathcal{L}\left(E_{1}, E_{0}\right): T \text { is bijective }\right\} .
$$

\footnotetext{
${ }^{6}$ A good summary of vector-valued Besov spaces and interpolation properties of these spaces can be found in [Am97], Chapter 5 and [Lu95], Chapter 1.
} 
Proposition 3.3. Let $M>0, r \geq 0, \theta \in[0,2 \pi]$ be constants,

$$
S t_{\theta, r}:=\left\{\lambda=\mu e^{i \theta}: \mu \geq r\right\}
$$

a ray in $\mathbb{C}$, and set

$$
\begin{aligned}
\mathcal{A}_{\theta, M}:= & \left\{A \in \mathcal{L}\left(E_{1}, E_{0}\right):(\lambda I-A) \in \mathcal{L}_{i s}\left(E_{1}, E_{0}\right)\right. \text { and } \\
& \left.\sum_{j=0}^{1}(1+|\lambda|)^{1-j}\|R(\lambda, A)\|_{\mathcal{L}\left(E_{0}, E_{j}\right)} \leq M \text { for all } \lambda \in S t_{\theta, r}\right\} .
\end{aligned}
$$

Then there exist constants $\varepsilon_{\theta}:=\varepsilon(\theta, M)>0, r_{\theta}>0$ and $C_{4}:=C_{4}(M):=$ $\frac{M}{M+1} \max \left\{2 M+3,4 M^{2}+7 M+1\right\}$ such that $\Sigma_{\theta, r_{\theta}}^{\varepsilon_{\theta}} \subset \rho(A)$ holds for all $A \in \mathcal{A}_{\theta, M}$ where

$$
\Sigma_{\theta, r_{\theta}}^{\varepsilon_{\theta}}:=\left\{\lambda \in \mathbb{C}:|\lambda| \geq r_{\theta} \text { and } \arg (\lambda) \in\left[\theta-\varepsilon_{\theta}, \theta+\varepsilon_{\theta}\right]\right\} .
$$

Moreover,

$$
\sum_{j=0}^{1}(1+|\lambda|)^{1-j}\|R(\lambda, A)\|_{\mathcal{L}\left(E_{0}, E_{j}\right)} \leq C_{4} \quad \text { for all } \lambda \in \Sigma_{\theta, r_{\theta}}^{\varepsilon_{\theta}} \text { and } A \in \mathcal{A}_{\theta, M} .
$$

Proof. First we will show two assertions.

Assertion I: Let $\theta=0$ in the hypotheses. Then there exist constants $\varepsilon_{0}>0, r_{0}>r$ and $\widetilde{M}:=\frac{M(2 M+3)}{M+1}$ so that $\Sigma_{0, r_{0}}^{\varepsilon_{0}} \subset \rho(A)$ for all $A \in \mathcal{A}_{0, M}$ and

$$
\|R(\lambda, A)\|_{\mathcal{L}\left(E_{0}\right)} \leq \frac{\widetilde{M}}{1+|\lambda|} \quad \forall \lambda \in \Sigma_{0, r_{0}}^{\varepsilon_{0}} \text { and } A \in \mathcal{A}_{0, M}
$$

Proof of Assertion I: We select a $r_{0}>r, \varepsilon_{0}:=\arcsin \left(\frac{1}{2(M+1)}\right)$ and define

$$
\Sigma_{\varepsilon_{0}, r_{0}}^{*}:=\left\{\lambda \in \mathbb{C}: \operatorname{Re} \lambda \geq r_{0} \text { and }|\arg (\lambda)| \leq \varepsilon_{0}\right\}
$$

and

$$
s:=\frac{|\lambda|^{2}}{\operatorname{Re} \lambda} \quad \text { for } \lambda \in \Sigma_{\varepsilon_{0}, r_{0}}^{*} .
$$

Then it follows from $|\lambda|=|i \operatorname{Re} \lambda-\operatorname{Im} \lambda|$ and $\lambda-s=\frac{\operatorname{Im} \lambda[i \operatorname{Re} \lambda-\operatorname{Im} \lambda]}{\operatorname{Re} \lambda}$ that for all $\lambda \in \Sigma_{\varepsilon_{0}, r_{0}}^{*}$ :

$\frac{1}{s}|\lambda-s|=\frac{\operatorname{Re} \lambda}{|\lambda|^{2}} \frac{|\operatorname{Im} \lambda||i \operatorname{Re} \lambda-\operatorname{Im} \lambda|}{\operatorname{Re} \lambda}=\left|\frac{\operatorname{Im} \lambda}{|\lambda|}\right|=|\sin (\arg (\lambda))| \leq \frac{1}{2(M+1)}$

(Note that $\varepsilon_{0} \in\left(0, \frac{\pi}{6}\right)$ and $f(t)=\sin (t)$ is increasing in $\left(-\frac{\pi}{6}, \frac{\pi}{6}\right)$ ). Thus

$$
|\lambda-s| \leq \frac{1+s}{2(M+1)} \quad \text { for all } \lambda \in \Sigma_{\varepsilon_{0}, r_{0}}^{*} .
$$

It is clear due to the hypotheses that $\left\{s \in \mathbb{R}: s \geq r_{0}\right\} \subset S t_{0, r} \subset \rho(A)$ for all $A \in \mathcal{A}_{0, M}$. For all $\lambda \in \Sigma_{\varepsilon_{0}, r_{0}}^{*}$ and $s$ as in (29) we get that

$$
\left\|(\lambda-s)(s I-A)^{-1}\right\|_{\mathcal{L}\left(E_{0}\right)} \leq|\lambda-s| \frac{M}{1+s} \leq \frac{1}{2} \text { for all } A \in \mathcal{A}_{0, M} .
$$

From this and a Neumann series argument it follows that $B:=(I+(\lambda-$ $\left.s)(s I-A)^{-1}\right)^{-1} \in \mathcal{L}\left(E_{0}\right)$ and $\|B\|_{\mathcal{L}\left(E_{0}\right)} \leq 2$. On the other hand we have that

$$
\lambda I-A=(s I-A)\left[I+(\lambda-s)(s I-A)^{-1}\right]=(s I-A) B^{-1} .
$$


Therefore $(\lambda I-A)^{-1} \in \mathcal{L}\left(E_{0}\right)$ and

$$
\begin{aligned}
\|R(\lambda, A)\|_{\mathcal{L}\left(E_{0}\right)} & \leq\|B\|_{\mathcal{L}\left(E_{0}\right)}\|R(s, A)\|_{\mathcal{L}\left(E_{0}\right)} \\
& \leq \frac{2 M}{1+s} \\
& =\frac{2 M}{1+|\lambda|} \frac{|\lambda-s+s|+1}{1+s} \\
& \leq \frac{2 M}{1+|\lambda|}\left[\frac{|\lambda-s|}{1+s}+1\right] \\
& \leq \frac{\widetilde{M}}{1+|\lambda|} \text { for all } \lambda \in \Sigma_{\varepsilon_{0}, r_{0}}^{*} \text { and } A \in \mathcal{A}_{0, M}
\end{aligned}
$$

with $\widetilde{M}:=\frac{M(2 M+3)}{M+1}$. Choosing $\widetilde{r}_{0} \geq r_{0}$ appropriately, we obtain a sector $\Sigma_{0, \widetilde{r}_{0}}^{\varepsilon_{0}} \subset \Sigma_{\varepsilon_{0}, r_{0}}^{*}$ which finishes the proof of Assertion I.

Assertion II: An analogous result to Assertion I also is valid if the hypotheses are fulfilled for a $\theta \in(0,2 \pi)$.

Proof of Assertion $I I$ : Let $\theta \in(0,2 \pi)$ and $\mathcal{O}: \mathbb{C} \rightarrow \mathbb{C}$ defined by $\mathcal{O}(z):=$ $e^{-i \theta} z, z \in \mathbb{C}$, a rotation function. It is clear that $\mathcal{O}$ is bijective and $\mathcal{O}^{-1}(z)=$ $e^{i \theta} z, z \in \mathbb{C}$. By hypotheses one knows that $S t_{\theta, r} \subset \rho(A)$ for all $A \in \mathcal{A}_{\theta, M}$ and furthermore

$$
\|R(\lambda, A)\|_{\mathcal{L}\left(E_{0}\right)} \leq \frac{M}{1+|\lambda|} \text { for all } \lambda \in S t_{\theta} \text { and } A \in \mathcal{A}_{\theta, M} .
$$

Therefore $e^{-i \theta} A \in \mathcal{A}_{0, M}$ for all $A \in \mathcal{A}_{\theta, M}$, because $R\left(\mu, e^{-i \theta} A\right)=e^{i \theta} R(\lambda, A)$ for all $\lambda=\mu e^{i \theta} \in S t_{\theta} \subset \rho(A)$, and

$\sum_{j=0}^{1}(1+|\mu|)^{1-j}\left\|R\left(\mu, e^{-i \theta} A\right)\right\|_{\mathcal{L}\left(E_{0}, E_{j}\right)}=\sum_{j=0}^{1}(1+|\lambda|)^{1-j}\|R(\lambda, A)\|_{\mathcal{L}\left(E_{0}, E_{j}\right)} \leq M$

for all $\lambda \in S t_{\theta, r}$ and $A \in \mathcal{A}_{\theta, M}$. Thus, we get from the Assertion I that

$$
\left\|R\left(w, e^{-i \theta} A\right)\right\|_{\mathcal{L}\left(E_{0}\right)} \leq \frac{\widetilde{M}}{1+|w|} \quad \text { for all } w \in \Sigma_{0, r_{0}}^{\varepsilon_{0}} \text { and } A \in \mathcal{A}_{\theta, M} .
$$

Now, let $\Sigma_{\theta, r_{\theta}}^{\varepsilon_{\theta}}:=\mathcal{O}^{-1}\left(\Sigma_{0, r_{0}}^{\varepsilon_{0}}\right)$. Then for each $\lambda \in \Sigma_{\theta, r_{\theta}}^{\varepsilon_{\theta}}$ (and hence $e^{-i \theta} \lambda \in$ $\left.\Sigma_{0, r_{0}}^{\varepsilon_{0}}\right)$ and each $A \in \mathcal{A}_{\theta, M}$ it holds that $R(\lambda, A)=e^{-i \theta} R\left(e^{-i \theta} \lambda, e^{-i \theta} A\right) \in$ $\mathcal{L}\left(E_{0}\right)$. Then $\Sigma_{\theta, r_{\theta}}^{\varepsilon_{\theta}} \subset \rho(A)$ and

$$
\|R(\lambda, A)\|_{\mathcal{L}\left(E_{0}\right)} \leq \frac{\widetilde{M}}{1+|\lambda|} \quad \text { for all } \lambda \in \Sigma_{\theta, r_{\theta}}^{\varepsilon_{\theta}}, A \in \mathcal{A}_{\theta, M} .
$$

Consequently Assertion II is proven. In order to finish the proof of this proposition it is sufficient to show that

$$
\|R(\lambda, A)\|_{\mathcal{L}\left(E_{0}, E_{1}\right)} \leq C_{4} \text { for all } \lambda \in \Sigma_{\theta, r_{\theta}}^{\varepsilon_{\theta}} \text { and } A \in \mathcal{A}_{\theta, M} .
$$

There $C_{4}:=\max \{\widetilde{M}, M(1+2 \widetilde{M})\}$. For it, let $A \in \mathcal{A}_{\theta, M}, \lambda \in \Sigma_{\theta, r_{\theta}}^{\varepsilon_{\theta}}$ and $\lambda_{\theta}:=r_{\theta} e^{i \theta} \in S t_{\theta, r}$. From $\left|\lambda_{\theta}\right|=r_{\theta} \leq|\lambda|$ and

$$
x=\left(\lambda_{\theta}-A\right)^{-1}\left[(\lambda-A) x+\left(\lambda_{\theta}-\lambda\right) x\right] \quad \text { for all } x \in E_{1}
$$


it follows that

$$
\begin{aligned}
\|x\|_{E_{1}} & \leq\left\|R\left(\lambda_{\theta}, A\right)\right\|_{\mathcal{L}\left(E_{0}, E_{1}\right)}\left[\|(\lambda-A) x\|_{E_{0}}+\left|\lambda_{\theta}-\lambda\right|\|x\|_{E_{0}}\right] \\
& \left.\leq M\left[\|(\lambda-A) x\|_{E_{0}}+2|\lambda|\|x\|_{E_{0}}\right] \quad \text { (due to definition of } \mathcal{A}_{\theta, M}\right) .
\end{aligned}
$$

Moreover, it follows from Assertion II that

$$
\|R(\lambda, A) y\|_{E_{0}} \leq \frac{\widetilde{M}}{1+|\lambda|}\|y\|_{E_{0}} \text { for all } y \in E_{0}, A \in \mathcal{A}_{\theta, M} \text { and } \lambda \in \Sigma_{\theta, r_{\theta}}^{\varepsilon_{\theta}},
$$

and therefore for all $x \in E_{1}$ (and hence $(\lambda-A) x \in E_{0}$ ) we obtain

$$
\|x\|_{E_{0}}=\|R(\lambda, A)[(\lambda-A) x]\|_{E_{0}} \leq \frac{\widetilde{M}}{1+|\lambda|}\|(\lambda-A) x\|_{E_{0}}
$$

for all $A \in \mathcal{A}_{\theta, M}, \lambda \in \Sigma_{\theta, r_{\theta}}^{\varepsilon_{\theta}}$. From this and (32) we get

$$
\begin{aligned}
\|x\|_{E_{1}} & \leq M\left[\|(\lambda-A) x\|_{E_{0}}+2 \widetilde{M} \frac{|\lambda|}{1+|\lambda|}\|(\lambda-A) x\|_{E_{0}}\right] \\
& \leq M(1+2 \widetilde{M})\|(\lambda-A) x\|_{E_{0}}
\end{aligned}
$$

for all $x \in E_{1}, A \in \mathcal{A}_{\theta, M}$ and $\lambda \in \Sigma_{\theta, r_{\theta}}^{\varepsilon_{\theta}}$. As $(\lambda-A) \in \mathcal{L}_{i s}\left(E_{1}, E_{0}\right)$, this yields the assertion (31).

Definition 3.4. Let $m \in \mathbb{R}^{+}, \rho \in \mathbb{N}_{0}$ and $a \in S_{1,0}^{m, \rho}\left(\mathbb{R}^{n}, \mathcal{L}\left(E_{1}, E_{0}\right)\right)$. Then the symbol $a$ is called parabolic in $S_{1,0}^{m, \rho}\left(\mathbb{R}^{n}, \mathcal{L}\left(E_{1}, E_{0}\right)\right)$ with constants $\omega$ and $\kappa$, if there are constants $\omega \geq 0$ and $\kappa>0$, so that for all $(\xi, \mu) \in \mathbb{R}^{n} \times \mathbb{R}_{0}^{+}$ with $|\xi, \mu| \geq \omega$ and $\theta \in\left[-\frac{\pi}{2}, \frac{\pi}{2}\right], a(\xi)+\mu^{m} e^{i \theta} I: E_{1} \longrightarrow E_{0}$ is bijective,

$$
\left[a(\xi)+\mu^{m} e^{i \theta} I\right]^{-1} \in \mathcal{L}\left(E_{0}, E_{1}\right)
$$

and

$$
\left\|\left[a(\xi)+\mu^{m} e^{i \theta} I\right]^{-1}\right\|_{\mathcal{L}\left(E_{0}, E_{1}\right)} \leq \kappa\langle\xi, \mu\rangle^{-m} .
$$

Here, $\langle\xi, \mu\rangle:=\sqrt{1+|\xi|^{2}+\mu^{2}}$ and $|\xi, \mu|:=\sqrt{|\xi|^{2}+\mu^{2}}$.

In the following proposition, we denote for $R \geq 0$ and $0<\theta \leq \pi$

$$
\Sigma_{\theta, R}:=\{\lambda \in \mathbb{C}:|\lambda| \geq R \text { and }|\arg (\lambda)| \leq \theta\},
$$

and in his proof we will use the inequality

$$
1+t^{m} \leq 2(1+t)^{m} \quad \text { for all } t \geq 0,
$$

where $m \geq 0$ (this inequality is obtained from $1 \leq(1+t)^{m}$ and $t^{m} \leq$ $(1+t)^{m}$ for all $\left.t \geq 0\right)$.

Proposition 3.5. Let $s \in \mathbb{R}, p, q \in[1, \infty], m \in \mathbb{R}^{+}, \rho_{n}$ as in (3) and $\mathcal{A} \subset S_{1,0}^{m, \rho_{n}}\left(\mathbb{R}^{n}, \mathcal{L}\left(E_{1}, E_{0}\right)\right)$ be bounded. Moreover, assume that all $a \in \mathcal{A}$ are parabolic in $S_{1,0}^{m, \rho_{n}}\left(\mathbb{R}^{n}, \mathcal{L}\left(E_{1}, E_{0}\right)\right)$ with the same constants $\omega \geq 0$ and $\kappa>0$, and $A:=\left.\widetilde{a(D)}\right|_{B_{p, q}^{s+m}\left(\mathbb{R}^{n}, E_{1}\right)}, a \in \mathcal{A}$. Then $\Sigma_{\frac{\pi}{2}, R} \subset \rho(-A)$ for $R=\omega^{m}$ and

$$
(1+|\lambda|)^{1-j}(\lambda+a)^{-1} \in S_{1,0}^{-j m, \rho_{n}}\left(\mathbb{R}^{n}, \mathcal{L}\left(E_{0}, E_{j}\right)\right), \quad j=0,1,
$$


PSEUDODIFFERENTIAL OPERATORS ON VECTOR-VALUED SOBOLEV SPACES 15

$$
\begin{gathered}
(1+|\lambda|)^{1-j}\left\|(\lambda+a)^{-1}\right\|_{S_{1,0}^{-j m, \rho_{n}}\left(\mathbb{R}^{n}, \mathcal{L}\left(E_{0}, E_{j}\right)\right)} \leq C_{5}, \quad j=0,1, \\
(1+|\lambda|)^{1-j}\left\|(\lambda I+A)^{-1}\right\|_{\mathcal{L}\left(B_{p, q}^{s}\left(\mathbb{R}^{n}, E_{0}\right), B_{p, q}^{s+j m}\left(\mathbb{R}^{n}, E_{j}\right)\right)} \leq C_{6}, \quad j=0,1
\end{gathered}
$$

for all $\lambda \in \Sigma_{\frac{\pi}{2}, R}$ and all $a \in \mathcal{A}$, where the constants

$$
C_{5}:=\kappa\left(\kappa \sup _{a \in \mathcal{A}}\|a\|_{S_{1,0}^{m, \rho_{n}}}\right)^{n} \max _{|\alpha| \leq \rho_{n}}\left\{\sum_{J=1}^{|\alpha|} \sum_{\substack{\alpha_{1}+\cdots+\alpha_{J}=\alpha \\ \alpha_{1}, \cdots, \alpha_{J} \neq 0}} 1\right\} \quad \text { und } \quad C_{6}:=C_{3} C_{5}
$$

are independent of $\lambda$ and a. Furthermore,

$$
(\lambda I+A)^{-1}=\left.\widetilde{b_{\lambda}(D)}\right|_{B_{p, q}^{s}\left(\mathbb{R}^{n}, E_{0}\right)} \quad \text { for all } \lambda \in \Sigma_{\frac{\pi}{2}, R} \text { and } a \in \mathcal{A},
$$

where $b_{\lambda}(\xi):=(\lambda+a(\xi))^{-1}, \xi \in \mathbb{R}^{n}$.

Proof. This follows from Theorem 7.2 in [Am97].

Propositions 3.3 and 3.5 imply:

Corollary 3.6. Let $s \in \mathbb{R}, p, q \in[1, \infty], m \in \mathbb{R}^{+}, \mathcal{B} \in\{B, \stackrel{\circ}{B}, b\}$ and $\mathcal{A} \subset$ $S_{1,0}^{m, \rho_{n}}\left(\mathbb{R}^{n}, \mathcal{L}\left(E_{1}, E_{0}\right)\right)$ be bounded. Moreover, assume that all $a \in \mathcal{A}$ are parabolic with the same constants $\omega \geq 0$ and $\kappa>0$, and $A:=\left.\widetilde{a(D)}\right|_{\mathcal{B}_{p, q}^{s+m}\left(\mathbb{R}^{n}, E_{1}\right)}$, $a \in \mathcal{A}$. Then, there exist constants ${ }^{7} C_{9}>0, C_{9}=C_{4}\left(C_{6}\right), 0<\vartheta<\pi / 2$ and $R>0$, so that ${ }^{8} \sum_{\frac{\pi}{2}+\vartheta, R} \subset \rho(-A)$ and

$$
\left\|(\lambda I+A)^{-1}\right\|_{\mathcal{L}\left(\mathcal{B}_{p, q}^{s}\left(\mathbb{R}^{n}, E_{0}\right)\right)} \leq \frac{C_{9}}{1+|\lambda|} \quad \text { for all } \lambda \in \Sigma_{\frac{\pi}{2}+\vartheta, R}, a \in \mathcal{A},
$$

where $C_{9}$ is independent of $\lambda$ and $a$. Therefore, the operator

$$
-A: \mathcal{B}_{p, q}^{s+m}\left(\mathbb{R}^{n}, E_{1}\right) \subset \mathcal{B}_{p, q}^{s}\left(\mathbb{R}^{n}, E_{0}\right) \longrightarrow \mathcal{B}_{p, q}^{s}\left(\mathbb{R}^{n}, E_{0}\right)
$$

generates an analytic semigroup ${ }^{9}$ on $\mathcal{B}_{p, q}^{s}\left(\mathbb{R}^{n}, E_{0}\right)$ for each $a \in \mathcal{A}$. Furthermore, $-A$ generates a strongly continuous semigroup on $\mathcal{B}_{p, q}^{s}\left(\mathbb{R}^{n}, E_{0}\right)$, if $E_{1} \stackrel{d}{\hookrightarrow} E_{0}$ and $q<\infty$.

Definition 3.7 (the realization of an operator). Let $A: D(A) \subset E_{0} \rightarrow E_{0}$ a linear operator and $E_{1} \hookrightarrow E_{0}$. Then one defines the $E_{1}-$ realization of $A$ (denoted with $A_{E_{1}}$ ) by

$$
D\left(A_{E_{1}}\right):=\left\{x \in D(A) \cap E_{1}: A x \in E_{1}\right\}, \quad A_{E_{1}} x:=A x, x \in D\left(A_{E_{1}}\right) .
$$

\footnotetext{
${ }^{7}$ For the definition of $C_{9}=C_{4}\left(C_{6}\right)$ see Propositions 3.3 and 3.5

${ }^{8}$ In this case, it is understood $A: D(A) \subset E \longrightarrow E$ with $D(A)=\mathcal{B}_{p, q}^{s+m}\left(\mathbb{R}^{n}, E_{1}\right)$ and $E=\mathcal{B}_{p, q}^{s}\left(\mathbb{R}^{n}, E_{0}\right)$.

${ }^{9}$ We took the definitions of analytic and strongly continuous semigroup from [Lu,95] chapter 2 .
} 
If the operator $A$ is closed, then the realization

$$
A_{E_{1}}: D\left(A_{E_{1}}\right) \subset E_{1} \longrightarrow E_{1}
$$

is closed, too. In this case, $\left(D\left(A_{E_{1}}\right),\|\cdot\|_{D\left(A_{E_{1}}\right)}\right)$ is a Banach space with the graph norm:

$$
\|u\|_{D\left(A_{E_{1}}\right)}:=\|u\|_{E_{1}}+\left\|A_{E_{1}} u\right\|_{E_{1}}, \quad u \in D\left(A_{E_{1}}\right) .
$$

We will show in the following that a suitable realization of the operator $-\widetilde{a(D)}$ generates a $C^{\infty}$-semigroup on $W_{p}^{k}\left(\mathbb{R}^{n}, E_{0}\right), B U C^{k}\left(\mathbb{R}^{n}, E_{0}\right)$, $C_{0}^{k}\left(\mathbb{R}^{n}, E_{0}\right)$ and on $C_{b}^{k}\left(\mathbb{R}^{n}, E_{0}\right)$. For this purpose, we introduce the following notation

$$
\mathcal{B}:=\left\{\begin{array}{lll}
\stackrel{\circ}{B}, & \text { if } \mathfrak{F}_{p}^{k}=W_{p}^{k}, & 1 \leq p<\infty, \\
B, & \text { if } \mathfrak{F}_{p}^{k}=W_{\infty}^{k}, & p=\infty, \\
b, & \text { if } \mathfrak{F}_{p}^{k}=B \cup C^{k}, & p=\infty, \\
\stackrel{\circ}{B}, & \text { if } \mathfrak{F}_{p}^{k}=C_{0}^{k}, & p=\infty, \\
B, & \text { if } \mathfrak{F}_{p}^{k}=C_{b}^{k}, & p=\infty .
\end{array}\right.
$$

From this and (13)-(17) follows that

$$
\mathcal{B}_{p, 1}^{k}\left(\mathbb{R}^{n}, E\right) \hookrightarrow \mathfrak{F}_{p}^{k}\left(\mathbb{R}^{n}, E\right) \hookrightarrow \mathcal{B}_{p, \infty}^{k}\left(\mathbb{R}^{n}, E\right), \quad 1 \leq p \leq \infty .
$$

On the basis of the above results and notations one obtains, similar to the proof of the Theorem 7.6 in [Am97], the following assertion:

Proposition 3.8. Let the hypotheses of the Proposition 3.5 be satisfied, $\alpha \in$ $(0,1), m>0, k \in \mathbb{N}_{0}, \mathfrak{F}_{p}^{k}=W_{p}^{k}$ for $1 \leq p<\infty, \mathfrak{F}_{\infty}^{k} \in\left\{W_{\infty}^{k}, B U C^{k}, C_{0}^{k}, C_{b}^{k}\right\}$ and $\mathcal{B}$ as in (40), and let now $A:=\left.\widetilde{a(D)}\right|_{\mathcal{B}_{p, \infty}^{k+m}\left(\mathbb{R}^{n}, E_{1}\right)}$ for $a \in \mathcal{A}$ and $A_{\mathfrak{F}_{p}^{k}}$ the $\mathfrak{F}_{p}^{k}\left(\mathbb{R}^{n}, E_{0}\right)$-realization of $A$. Then $-A_{\mathfrak{F}_{p}^{k}}$ generates a $C^{\infty}$-semigroup of singular type $\alpha$ on the space $\mathfrak{F}_{p}^{k}\left(\mathbb{R}^{n}, E_{0}\right)$. Strictly speaking, there are constants $M>0, \widetilde{\omega} \in \mathbb{R}$ and $\widetilde{\theta} \in] \frac{\pi}{2}, \pi[$, wich do not depend on a and $\lambda$, such that $\{\lambda \in \mathbb{C}: \lambda \neq \widetilde{\omega}$ and $|\arg (\lambda-\widetilde{\omega})|<\widetilde{\theta}\}=: S_{\widetilde{\theta}, \widetilde{\omega}} \subset \rho\left(-A_{\widetilde{F}_{p}^{k}}\right)$ and

$$
\left\|\left(\lambda I+A_{\mathfrak{F}_{p}^{k}}\right)^{-1}\right\|_{\mathcal{L}\left(\mathfrak{F}_{p}^{k}\left(\mathbb{R}^{n}, E_{0}\right)\right)} \leq \frac{M}{(1+|\lambda|)^{\alpha}}
$$

for all $\lambda \in S_{\widetilde{\theta}, \widetilde{\omega}}$ and $a \in \mathcal{A}$. Moreover, it holds

$$
\left(\lambda I+A_{\widetilde{F}_{p}^{k}}\right)^{-1}=\left.\left(\lambda I+\left.\widetilde{a(D)}\right|_{\mathcal{B}_{p, \infty}^{k+m}\left(\mathbb{R}^{n}, E_{1}\right)}\right)^{-1}\right|_{\mathfrak{F}_{p}^{k}\left(\mathbb{R}^{n}, E_{0}\right)} .
$$

Proof. First one shows equality (43) with the help of Proposition 3.5 and Corollary 3.6. Afterwards, one uses Proposition 3.5, the continuous embeddings

$$
\mathcal{B}_{p, \infty}^{k+m}\left(\mathbb{R}^{n}, E_{1}\right) \hookrightarrow \mathcal{B}_{p, 1}^{k+\delta}\left(\mathbb{R}^{n}, E_{0}\right) \hookrightarrow \mathfrak{F}_{p}^{k} \hookrightarrow \mathcal{B}_{p, \infty}^{k}\left(\mathbb{R}^{n}, E_{0}\right) \hookrightarrow \mathcal{B}_{p, 1}^{k-\varepsilon}\left(\mathbb{R}^{n}, E_{0}\right)
$$

for all $0<\varepsilon<\delta<m$ and $\mathfrak{F}_{p}^{k}:=\mathfrak{F}_{p}^{k}\left(\mathbb{R}^{n}, E_{0}\right)$, and interpolation properties of the Besov space to prove that

$$
\left\|\left(\lambda I+A_{\mathfrak{F}_{p}^{k}}\right)^{-1}\right\|_{\mathcal{L}\left(\mathfrak{F}_{p}^{k}\left(\mathbb{R}^{n}, E_{0}\right)\right)} \leq c(\varepsilon, \delta)(1+|\lambda|)^{\frac{\varepsilon-\delta}{\varepsilon+\delta}} \quad \text { for all } \lambda \in S_{\widetilde{\theta}, \widetilde{\omega}} \text { and } a \in \mathcal{A} \text {. }
$$


Setting $\varepsilon=\frac{(1-\alpha) \delta}{1+\alpha}$, we obtain (42).

Remark 3.9. Let $A$ be as in Proposition 3.8 and let

$$
D_{\max }(A):=\left\{u \in \mathfrak{F}_{p}^{k}\left(\mathbb{R}^{n}, E_{1}\right): A u \in \mathfrak{F}_{p}^{k}\left(\mathbb{R}^{n}, E_{0}\right)\right\} .
$$

Then $D_{\max }(A)=D\left(A_{\mathfrak{F}_{p}^{k}}\right)=\left\{u \in \mathcal{B}_{p, \infty}^{k+m}\left(\mathbb{R}^{n}, E_{1}\right): A u \in \mathfrak{F}_{p}^{k}\left(\mathbb{R}^{n}, E_{0}\right)\right\}$.

Proof. It is clear that $D\left(A_{\mathfrak{F}_{p}^{k}}\right) \subset D_{\max }(A)$, because $\mathcal{B}_{p, \infty}^{k+m}\left(\mathbb{R}^{n}, E_{1}\right) \hookrightarrow \mathfrak{F}_{p}^{k}\left(\mathbb{R}^{n}, E_{1}\right)$. Now, let $u \in D_{\max }(A)$, then $u, A u \in \mathfrak{F}_{p}^{k}\left(\mathbb{R}^{n}, E_{0}\right)$ und due to $\mathfrak{F}_{p}^{k}\left(\mathbb{R}^{n}, E\right) \hookrightarrow$ $\mathcal{B}_{p, \infty}^{k}\left(\mathbb{R}^{n}, E\right), v:=(\lambda+A) u \in \mathcal{B}_{p, \infty}^{k}\left(\mathbb{R}^{n}, E_{0}\right)$. Let $\lambda \in \mathbb{C}$ such that

$$
\lambda+A: \mathcal{B}_{p, \infty}^{k+m}\left(\mathbb{R}^{n}, E_{1}\right) \longrightarrow \mathcal{B}_{p, \infty}^{k}\left(\mathbb{R}^{n}, E_{0}\right) \text { is bijective }
$$

(see Corollary 3.6). Then $u=(\lambda+A)^{-1} v \in \mathcal{B}_{p, \infty}^{k+m}\left(\mathbb{R}^{n}, E_{1}\right)$ and thus $u \in$ $D\left(A_{\mathfrak{F}_{p}^{k}}\right)$.

Now, we ask ourselves whether $-A_{\mathfrak{F}_{p}^{k}}$ generates also an analytic semigroup on $\mathfrak{F}_{p}^{k}\left(\mathbb{R}^{n}, E_{0}\right)$. For parabolic symbols $a$ in $S_{1,0}^{m, \rho_{n}}\left(\mathbb{R}^{n}, \mathcal{L}\left(E_{1}, E_{0}\right)\right)$ we will show in Theorem 3.14 and Corollary 3.15, that the $\mathfrak{F}_{p}^{k}$-realization of the operator $-\widetilde{a(D)}$ generates an analytic semigroup on the spaces $\mathfrak{F}_{p}^{k}\left(\mathbb{R}^{n}, E_{0}\right)$. Generation of analytic semigroup on $L_{p}\left(\mathbb{R}^{n}, E_{0}\right), C_{0}\left(\mathbb{R}^{n}, E_{0}\right)$ and $B U C\left(\mathbb{R}^{n}, E_{0}\right)$ was proven by $\mathrm{H}$. Amann in [Am01], but this referred to $\mathcal{L}\left(E_{0}\right)$-vector valued and parabolic differential operators. In [Ki01] was proven the generation of analytic semigroup on $L_{p}\left(\mathbb{R}^{n}, E_{0}\right)(1 \leq p<\infty)$ by suitable parabolic pseudodifferential operators with $\mathcal{L}\left(E_{0}\right)$-valued symbols of order $m>1$, which have homogeneous principal part and in the dual variable $\xi$ regularity greather than or equal to $2 n+1$.

Lemma 3.10. Let $\mathcal{A}$ be a family of parabolic symbols in $S_{1,0}^{m, \rho}\left(\mathbb{R}^{n}, \mathcal{L}\left(E_{1}, E_{0}\right)\right)$ with the same constants $\kappa>0$ and $\omega \geq 0$, and let $\mathcal{A} \subset S_{1,0}^{m, \rho}\left(\mathbb{R}^{n}, \mathcal{L}\left(E_{1}, E_{0}\right)\right)$ be bounded, i.e. there is a constant $K>0$ with

$$
\|a\|_{S_{1,0}^{m, \rho}}<K \text { for all } a \in \mathcal{A} \text {. }
$$

Then, for $R:=\omega^{m}$ and $b_{\lambda}:=(\lambda+a)^{-1}$ we have the estimate

$$
\left\|\partial_{\xi}^{\alpha} b_{\lambda}(\xi)\right\|_{\mathcal{L}\left(E_{0}\right)} \leq C_{10}\langle\xi\rangle^{m-|\alpha|}\left\langle\xi,|\lambda|^{1 / m}\right\rangle^{-2 m}
$$

for all $\xi \in \mathbb{R}^{n}, \lambda \in \Sigma_{\frac{\pi}{2}, R}, a \in \mathcal{A}$ and $0<|\alpha| \leq \rho$, where $C_{10}:=\rho C_{5}$ (see Proposition 3.5).

Remark 3.11. It was shown in [Ba09] that the set $\left\{b_{\lambda}: a \in \mathcal{A}, \lambda \in \Sigma_{\frac{\pi}{2}, R}\right\}$ is bounded in $S_{1,0}^{-m, \rho}\left(\mathbb{R}^{n}, \mathcal{L}\left(E_{0}, E_{1}\right)\right)$.

Proof of Lemma 3.10. Let $\beta \in \mathbb{N}_{0}^{n}$ with $|\beta| \leq \rho$ and $\left(|\lambda|^{\frac{1}{m}}\right)^{m} e^{i \arg (\lambda)}=\lambda \in$ $\Sigma_{\frac{\pi}{2}, R}$, then

$$
\begin{aligned}
\left\|\left(\partial_{\xi}^{\beta} a(\xi)\right)(\lambda+a(\xi))^{-1}\right\|_{\mathcal{L}\left(E_{0}\right)} & \leq\left\|\partial_{\xi}^{\beta} a(\xi)\right\|_{\mathcal{L}\left(E_{1}, E_{0}\right)}\left\|(\lambda+a(\xi))^{-1}\right\|_{\mathcal{L}\left(E_{0}, E_{1}\right)} \\
& \leq\|a\|_{S_{1,0}^{m, \rho}}\langle\xi\rangle^{m-|\beta|} \kappa\left\langle\xi,|\lambda|^{1 / m}\right\rangle^{-m}
\end{aligned}
$$


Now, let $\alpha \in \mathbb{N}_{0}^{n}$ with $0<|\alpha| \leq \rho$. Since $\partial_{\xi}^{\alpha}(\lambda+a)^{-1}$ is a sum of terms of the form

$$
\pm(\lambda+a)^{-1}\left(\partial_{\xi}^{\alpha_{1}} a\right)(\lambda+a)^{-1} \ldots\left(\partial_{\xi}^{\alpha_{k}} a\right)(\lambda+a)^{-1}
$$

with $\alpha_{i} \in \mathbb{N}_{0}^{n} \backslash\{0\}, \alpha_{1}+\ldots+\alpha_{j}=\alpha$ and $j=1, . .,|\alpha|$, it follows from (48) that

$$
\begin{aligned}
& \left\|\partial_{\xi}^{\alpha}(\lambda+a(\xi))^{-1}\right\|_{\mathcal{L}\left(E_{0}\right)} \\
& \leq\left\|(\lambda+a(\xi))^{-1}\right\|_{\mathcal{L}\left(E_{0}\right)} \sum_{\substack{j=1 \\
j=1}} \sum_{\substack{\alpha_{1}+\ldots+\alpha_{j}=\alpha \\
\alpha_{1}, \cdots, \alpha_{j} \neq 0}} \prod_{i=1}^{j}\left\|\left(\partial_{\xi}^{\alpha_{i}} a(\xi)\right)(\lambda+a(\xi))^{-1}\right\|_{\mathcal{L}\left(E_{0}\right)} \\
& \leq \kappa\left\langle\xi,|\lambda|^{1 / m}\right\rangle^{-m} \sum_{j=1}^{|\alpha|} \sum_{\substack{\alpha_{1}+\ldots, \alpha_{j}=\alpha \\
\alpha_{1}, \cdots, \alpha_{j} \neq 0}} \prod_{i=1}^{j} \kappa\|a\|_{S_{1,0}^{m, \rho_{n}}}\langle\xi\rangle^{m-\left|\alpha_{i}\right|}\left\langle\xi,|\lambda|^{1 / m}\right\rangle^{-m} \\
& \leq C_{5} \sum_{j=1}^{|\alpha|}\langle\xi\rangle^{j m-|\alpha|}\left\langle\xi,|\lambda|^{1 / m}\right\rangle^{-m(j+1)} \\
& \leq C_{5}\langle\xi\rangle^{m-|\alpha|}\left\langle\xi,|\lambda|^{1 / m}\right\rangle^{-2 m} \sum_{j=1}^{|\alpha|} \underbrace{\langle\xi\rangle^{m(j-1)}\left\langle\xi,|\lambda|^{1 / m}\right\rangle^{m(1-j)}}_{\leq 1} \\
& \leq \rho C_{5}\langle\xi\rangle^{m-|\alpha|}\left\langle\xi,|\lambda|^{1 / m}\right\rangle^{-2 m}
\end{aligned}
$$

for all $\xi \in \mathbb{R}^{n}, \lambda \in \Sigma_{\frac{\pi}{2}, R}$ and $a \in \mathcal{A}$ which finishes the proof of the lemma.

In the following section non-autonomous evolution equations will be considered and therefore we will show the validity of the following proposition for a family of parabolic symbols. But before we mention an elementary lemma.

Lemma 3.12. Let $\chi \in \mathcal{S}\left(\mathbb{R}^{n}\right)$ with $\chi(0)=1$. Then:

a) $\chi(\varepsilon x) \underset{\varepsilon \rightarrow 0}{\longrightarrow} 1$ uniformly on all compact subset of $\mathbb{R}^{n}$.

b) $\partial_{x}^{\alpha} \chi(\varepsilon x) \underset{\varepsilon \rightarrow 0}{\longrightarrow} 0$ uniformly on $\mathbb{R}^{n}$, if $\alpha \neq 0$.

c) For all $\alpha \in \mathbb{N}_{0}^{n}$ there is a $c_{\alpha}>0$, independent of $0<\varepsilon<1$, such that

$$
\left|\partial_{x}^{\alpha} \chi(\varepsilon x)\right| \leq c_{\alpha} \varepsilon^{\sigma}\langle x\rangle^{-(|\alpha|-\sigma)}, \quad(0 \leq \sigma \leq|\alpha|), \text { for all } x \in \mathbb{R}^{n} .
$$

Proof. See [Ku81], Lemma 6.3.

In Lemma 3.12 we can choose $c_{\alpha}=2^{\frac{|\alpha|-\sigma}{2}}\|\chi\|_{|\alpha|-\sigma,|\alpha|}$, where

$$
\|\chi\|_{k, l}:=\max _{|\beta| \leq k} \sup _{\xi \in \mathbb{R}^{n}}\langle\xi\rangle^{k}\left|\partial_{\xi}^{\beta} \chi(\xi)\right|, \quad k, l \in \mathbb{N}_{0},
$$


is the standard seminorm in $\mathcal{S}\left(\mathbb{R}^{n}\right)$. In the following Proposition

$$
\begin{aligned}
& C_{11}:=2^{\frac{|\alpha|}{2}}\|\chi\|_{|\alpha|,|\alpha|}, \quad C_{12}:=\rho_{n} C_{5} \max _{|\alpha| \leq n+1}\left\{C_{11} \sum_{\gamma \leq \alpha}\left(\begin{array}{l}
\alpha \\
\gamma
\end{array}\right)\right\}, \\
& C_{13}:=2 n \omega_{n} C_{12} \max \left\{\frac{2 m}{m^{2}-\theta_{0}^{2}}, \frac{2 m}{m^{2}-\left(1-\theta_{1}\right)^{2}}, \frac{1}{m-\theta_{0}}+\frac{1}{n}\right\},
\end{aligned}
$$

where $0<\theta_{i}<1$ for $i=0,1, C^{*}:=n^{\frac{n+1}{2}}\left(\sum_{|\alpha|=n+1} 1\right) C_{13}$,

$$
\hat{C}:=C^{*}\left\|\frac{|\cdot|^{\theta_{0}-n}+|\cdot|^{\theta_{1}-n}}{1+|\cdot|}\right\|_{L_{1}} \quad \text { and } \quad M_{1}:=\frac{2 \hat{C}}{(2 \pi)^{n}} .
$$

Proposition 3.13. Let $m>0,1 \leq p \leq \infty, \mathcal{A} \subset S_{1,0}^{m, \rho_{n}}\left(\mathbb{R}^{n}, \mathcal{L}\left(E_{1}, E_{0}\right)\right)$ be bounded and $k \in \mathbb{N}_{0}$. Moreover, we suppose that all symbols $a \in \mathcal{A}$ are parabolic with the same constants $\omega \geq 0$ and $\kappa>0$. Then there exist constants $R>0$ and $0<\theta_{i}<1, i=0,1$, such that

$$
\left\|\left.\widetilde{b_{\lambda}(D)}\right|_{\mathcal{B}_{p, \infty}^{k}\left(\mathbb{R}^{n}, E_{0}\right)} u\right\|_{W_{p}^{k}\left(\mathbb{R}^{n}, E_{0}\right)} \leq \frac{M_{1}}{1+|\lambda|}\|u\|_{W_{p}^{k}\left(\mathbb{R}^{n}, E_{0}\right)}
$$

for all $u \in C_{b}^{\infty}\left(\mathbb{R}^{n}, E_{0}\right) \cap W_{p}^{k}\left(\mathbb{R}^{n}, E_{0}\right), \lambda \in \sum_{\frac{\pi}{2}, R}$ and $a \in \mathcal{A}$. There, $b_{\lambda}=$ $(\lambda+a)^{-1}, \mathcal{B}:=\stackrel{\circ}{B}$ if $1 \leq p<\infty$ and $\mathcal{B}:=B$ if $p=\infty$.

Proof. We can suppose without loss of generality that $R \geqslant 1$ in Remark 3.10. By definition of $\left\|\left.\widetilde{b_{\lambda}(D)}\right|_{\mathcal{B}_{p, \infty}^{k}\left(\mathbb{R}^{n}, E_{0}\right)} u\right\|_{W_{p}^{k}\left(\mathbb{R}^{n}, E_{0}\right)}$ we have to calculate the derivatives $\partial_{x}^{\beta}\left(\widetilde{b_{\lambda}(D)} u(x)\right)$. From the formulas about differentiation under the integral and transformation of oscillatory integrals it follows that for all $u \in C_{b}^{\infty}\left(\mathbb{R}^{n}, E_{0}\right) \cap W_{p}^{k}\left(\mathbb{R}^{n}, E_{0}\right), \lambda \in \Sigma_{\frac{\pi}{2}, R},|\lambda|:=\mu^{m}, \hat{\lambda}:=\mu^{-m} \lambda$ and $|\beta| \leq k$ :

$$
\begin{aligned}
& \partial_{x}^{\beta}\left(\widetilde{b_{\lambda}(D)} u(x)\right) \\
& =o s-\iint e^{i \xi \cdot \eta} b_{\lambda}(\xi) \partial_{x}^{\beta} u(x-\eta) \frac{d(\xi, \eta)}{(2 \pi)^{n}} \\
& =o s-\iint e^{i \xi \cdot \eta} \mu^{-m}\left(\hat{\lambda}+\mu^{-m} a(\xi)\right)^{-1} \partial_{x}^{\beta} u(x-\eta) \frac{d(\xi, \eta)}{(2 \pi)^{n}} \\
& =\lim _{\varepsilon \rightarrow 0} \int_{\mathbb{R}^{n}}\left(\int_{\mathbb{R}^{n}} e^{i \xi \cdot \eta} \chi_{\varepsilon}(\xi, \eta)\left(\hat{\lambda}+\mu^{-m} a(\xi)\right)^{-1} d \xi\right) \partial_{x}^{\beta} u(x-\eta) \frac{d \eta}{|\lambda|(2 \pi)^{n}} .
\end{aligned}
$$

Thus

$$
\partial_{x}^{\beta}\left(\widetilde{b_{\lambda}(D)} u(x)\right)=\lim _{\varepsilon \rightarrow 0} \int_{\mathbb{R}^{n}} K_{\varepsilon}(\eta, \lambda) \partial_{x}^{\beta} u(x-\eta) \frac{d \eta}{|\lambda|(2 \pi)^{n}}
$$

with

$$
K_{\varepsilon}(\eta, \lambda):=\int_{\mathbb{R}^{n}} e^{i \xi \cdot \eta} \chi_{\varepsilon}(\xi, \eta)\left(\hat{\lambda}+\mu^{-m} a(\xi)\right)^{-1} d \xi
$$


and $\chi_{\varepsilon}(\xi, \eta):=\chi(\varepsilon \xi) \chi(\varepsilon \eta)$ for all $\xi, \eta \in \mathbb{R}^{n}, 0<\varepsilon<1, \chi$ is a function in $\mathcal{S}\left(\mathbb{R}^{n}\right)$ with $\chi \equiv 1$ on $|\xi| \leq 1$ and $\chi \equiv 0$ on $|\xi| \geq 2$. It observes that in (49) $\partial_{x}^{\beta} u \in L_{p}\left(\mathbb{R}^{n}, E_{0}\right)$ for all $|\beta| \leq k$. Now we will show the following assertion.

Assertion: i) There exist constants $0<\theta_{i}<1, i=0,1$, such that for all $\varepsilon \in(0,1), \eta \in \mathbb{R}^{n}, \lambda \in \sum_{\frac{\pi}{2}, R}\left(|\lambda|=\mu^{m}\right)$ and $a \in \mathcal{A}$ :

$$
(1+|\mu \eta|)|\mu \eta|^{n}\left\|K_{\varepsilon}(\eta, \lambda)\right\|_{\mathcal{L}\left(E_{0}\right)} \leq C^{*} \mu^{n}\left(|\mu \eta|^{\theta_{0}}+|\mu \eta|^{\theta_{1}}\right) .
$$

There, $C^{*}$ and $\theta_{i}$ are independent of $\varepsilon, \eta, \lambda$ and $a$.

ii) There is a strongly measurable function $K: \mathbb{R}^{n} \times \Sigma_{\frac{\pi}{2}, R} \longrightarrow \mathcal{L}\left(E_{0}\right)$ with

$$
K_{\varepsilon}(\eta, \lambda) \underset{\varepsilon \rightarrow 0}{\longrightarrow} K(\eta, \lambda) \text { pointwise for all }(\eta, \lambda) \in \mathbb{R}_{\eta}^{n} \times \sum_{\frac{\pi}{2}, R}
$$

and

$$
\|K(\cdot, \lambda)\|_{L_{1}\left(\mathbb{R}_{\eta}^{n}, \mathcal{L}\left(E_{0}\right)\right)} \leq \hat{C}
$$

for all $\lambda \in \Sigma_{\frac{\pi}{2}, R}$ and $a \in \mathcal{A}$, where the constant $\hat{C}>0$ is independent of $\lambda$ and $a$.

Proof of i):

$$
\begin{aligned}
K_{\varepsilon}(\eta, \lambda) & =\int_{\mathbb{R}^{n}} e^{i \xi \cdot \eta} \chi_{\varepsilon}(\xi, \eta)\left(\hat{\lambda}+\mu^{-m} a(\xi)\right)^{-1} d \xi \\
& \stackrel{\xi:=\mu \bar{\xi}}{=} \mu^{n} \int_{\mathbb{R}^{n}} e^{i \mu \bar{\xi} \cdot \eta} \chi_{\varepsilon}(\mu \bar{\xi}, \eta) \mu^{m}(\lambda+a(\mu \bar{\xi}))^{-1} d \bar{\xi} \\
& =\mu^{n+m} \int_{\mathbb{R}^{n}} e^{i \mu \xi \cdot \eta} \chi_{\varepsilon}(\mu \xi, \eta) b_{\lambda}(\mu \xi) d \xi
\end{aligned}
$$

Let $\alpha \in \mathbb{N}_{0}^{n}$ with $|\alpha|>0$. Then

$$
\int_{\mathbb{R}^{n}} D_{\xi}^{\alpha}\left(\chi_{\varepsilon}(\mu \xi, \eta) b_{\lambda}(\mu \xi)\right) d \xi=0
$$

and with this

$$
\begin{aligned}
\|(\mu \eta)^{\alpha} K_{\varepsilon} & (\eta, \lambda) \|_{\mathcal{L}\left(E_{0}\right)} \\
= & \left\|\mu^{n+m} \int_{\mathbb{R}^{n}} e^{i(\mu \xi) \cdot \eta} D_{\xi}^{\alpha}\left[\chi_{\varepsilon}(\mu \xi, \eta) b_{\lambda}(\mu \xi)\right] d \xi\right\| \\
& =\left\|\mu^{n+m} \int_{\mathbb{R}^{n}}\left(e^{i(\mu \xi) \cdot \eta}-1\right) D_{\xi}^{\alpha}\left[\chi_{\varepsilon}(\mu \xi, \eta) b_{\lambda}(\mu \xi)\right] d \xi\right\| \\
& \leq 2 \mu^{n+m} \int_{\mathbb{R}^{n}}|\mu \xi|^{\theta}|\eta|^{\theta}\left\|D_{\xi}^{\alpha}\left[\chi_{\varepsilon}(\mu \xi, \eta) b_{\lambda}(\mu \xi)\right]\right\| d \xi,
\end{aligned}
$$


because $\left|e^{i(\mu \xi) \cdot \eta}-1\right| \leq 2|\mu \xi|^{\theta}|\eta|^{\theta}$ for all $\xi, \eta \in \mathbb{R}^{n}$ and $0<\theta<1$. On the other hand, we obtain from Lemma 3.10 and Lemma 3.12:

$$
\begin{aligned}
\| D_{\xi}^{\alpha} & {\left[\chi_{\varepsilon}(\mu \xi, \eta) b_{\lambda}(\mu \xi)\right] \| } \\
& \leq \sum_{\gamma \leq \alpha}\left(\begin{array}{c}
\alpha \\
\gamma
\end{array}\right)|\chi(\varepsilon \eta)|\left|\partial_{\xi}^{\alpha-\gamma} \chi(\varepsilon \mu \xi)\right|\left\|\partial_{\xi}^{\gamma} b_{\lambda}(\mu \xi)\right\| \\
& \leq \sum_{\gamma \leq \alpha}\left(\begin{array}{c}
\alpha \\
\gamma
\end{array}\right) \rho_{n} C_{5} C_{11} \mu^{|\alpha|-|\gamma|}\langle\mu \xi\rangle^{|\gamma|-|\alpha|} \mu^{|\gamma|}\langle\mu \xi\rangle^{m-|\gamma|}\langle\mu \xi, \mu\rangle^{-2 m} \\
& \leq C_{12} \mu^{|\alpha|}\langle\mu \xi\rangle^{m-|\alpha|} \mu^{-2 m}|\xi, 1|^{-2 m} \\
& =C_{12} \mu^{|\alpha|}\langle\mu \xi\rangle^{m-|\alpha|} \mu^{-2 m}\langle\xi\rangle^{-2 m}
\end{aligned}
$$

for all $\xi, \eta \in \mathbb{R}^{n}, 0<\varepsilon<1, \lambda \in \sum_{\frac{\pi}{2}, R}$ and $a \in \mathcal{A}$. Then

$$
\left\|(\mu \eta)^{\alpha} K_{\varepsilon}(\eta, \lambda)\right\|_{\mathcal{L}\left(E_{0}\right)} \leq 2 C_{12} \mu^{n}|\mu \eta|^{\theta} \underbrace{\int_{\mathbb{R}^{n}}|\xi|^{\theta}\left(\sqrt{\frac{1}{\mu^{2}}+|\xi|^{2}}\right)^{m-|\alpha|}\langle\xi\rangle^{-2 m} d \xi}_{=: I(\theta,|\alpha|)}
$$

Now we show that there is $0<\theta_{i}<1$ such that $I\left(\theta_{i}, n+i\right)<\infty$ for $i=0,1$.

(1) We consider first the case $0<m<n$ :

$$
\begin{aligned}
I(\theta,|\alpha|) & =\int_{\mathbb{R}^{n}}|\xi|^{\theta}\left(\frac{1}{\mu^{2}}+|\xi|^{2}\right)^{\frac{m-|\alpha|}{2}}\langle\xi\rangle^{-2 m} d \xi \\
& \leq \int_{|\xi| \leq 1}|\xi|^{\theta}|\xi|^{m-|\alpha|} d \xi+\int_{|\xi| \geq 1}|\xi|^{\theta}|\xi|^{m-|\alpha|}|\xi|^{-2 m} d \xi \\
& =n \omega_{n}\left(\int_{0}^{1} r^{\theta} r^{m-|\alpha|} r^{n-1} d r+\int_{1}^{\infty} r^{\theta} r^{m-|\alpha|} r^{-2 m} r^{n-1} d r\right) \\
& =n \omega_{n}\left(\left.\frac{r^{\theta+m-|\alpha|+n}}{\theta+m-|\alpha|+n}\right|_{0} ^{1}+\left.\frac{r^{\theta-m-|\alpha|+n}}{\theta-m-|\alpha|+n}\right|_{1} ^{\infty}\right) .
\end{aligned}
$$

In case of $|\alpha|=n$ we can choose $\theta_{0} \in(0,1)$ such that $\theta_{0}<m$, because $m>0$. Then

$$
I\left(\theta_{0}, n\right) \leq n \omega_{n}\left(\left.\frac{r^{\theta_{0}+m}}{\theta_{0}+m}\right|_{0} ^{1}+\left.\frac{r^{\theta_{0}-m}}{\theta_{0}-m}\right|_{1} ^{\infty}\right)=\frac{2 n \omega_{n} m}{m^{2}-\theta_{0}^{2}}<\infty
$$

where $\omega_{n}$ denotes the volume of the unit ball in $\mathbb{R}^{n}$. In case of $|\alpha|=$ $n+1$ we can also choose $\theta_{1} \in(0,1)$ such that $m>1-\theta_{1}$. Then we have

$$
I\left(\theta_{1}, n+1\right) \leq n \omega_{n}\left(\left.\frac{r^{\theta_{1}+m-1}}{\theta_{1}+m-1}\right|_{0} ^{1}+\left.\frac{r^{\theta_{1}-m-1}}{\theta_{1}-m-1}\right|_{1} ^{\infty}\right)=\frac{2 n \omega_{n} m}{m^{2}-\left(1-\theta_{1}\right)^{2}}<\infty,
$$

because $\theta_{1}+m-1>0$ and $\theta_{1}-m-1=\left(\theta_{1}-1\right)-m<0$. Therefore for $0<m<n$ and $|\alpha|=n+i, i=0,1$, there are $\theta_{i} \in(0,1)$ and 
$C_{13}>0$, such that

$$
\left\|(\mu \eta)^{\alpha} K_{\varepsilon}(\eta, \lambda)\right\| \leq C_{13} \mu^{n}|\mu \eta|^{\theta_{i}}
$$

for all $\lambda \in \sum_{\frac{\pi}{2}, R}$ and $\eta \in \mathbb{R}^{n}$.

(2) Now we consider the case $m \geq n+1$ :

$$
\begin{aligned}
I(\theta,|\alpha|) & =\int_{\mathbb{R}^{n}}|\xi|^{\theta}\left(\frac{1}{\mu^{2}}+|\xi|^{2}\right)^{\frac{m-|\alpha|}{2}}\langle\xi\rangle^{-2 m} d \xi \\
& \left.\leq \int_{\mathbb{R}^{n}}\langle\xi\rangle^{\theta}\langle\xi\rangle^{m-|\alpha|}\langle\xi\rangle^{-2 m} d \xi \quad \text { (because } \mu \geq 1 \text { and } m-|\alpha| \geq 0\right) \\
& =\int_{\mathbb{R}^{n}}\langle\xi\rangle^{-(|\alpha|+m-\theta)} d \xi .
\end{aligned}
$$

We choose $\theta_{0} \in(0,1)$ such that $\theta_{0}<m$. Then we have $|\alpha|+m-$ $\theta_{0}>n$ and therefore $I\left(\theta_{0},|\alpha|\right)=n \omega_{n}\left(\frac{1}{n}+\frac{1}{m-\theta_{0}}\right)<\infty$. If we define $\theta_{1}:=\theta_{0}$, we have that there exists $\theta_{i} \in(0,1), i=0,1$, such that

$$
\left\|(\mu \eta)^{\alpha} K_{\varepsilon}(\eta, \lambda)\right\| \leq C_{13} \mu^{n}|\mu \eta|^{\theta_{i}}
$$

for all $\lambda \in \sum_{\frac{\pi}{2}, R}$ and $\eta \in \mathbb{R}^{n}$.

(3) Finally we consider the case $n \leq m<n+1$ : If $|\alpha|=n$ we can estimate like in the former case. Then we have to consider only $|\alpha|=$ $n+1$.

$$
\begin{aligned}
I(\theta, n+1) & =\int_{\mathbb{R}^{n}}|\xi|^{\theta}\left(\frac{1}{\mu^{2}}+|\xi|^{2}\right)^{\frac{m-(n+1)}{2}}\langle\xi\rangle^{-2 m} d \xi \\
& \leq \int_{|\xi| \leq 1}|\xi|^{\theta}|\xi|^{m-(n+1)} d \xi+\int_{|\xi| \geq 1}|\xi|^{\theta}|\xi|^{m-(n+1)}|\xi|^{-2 m} d \xi \\
& =n \omega_{n}\left(\int_{0}^{1} r^{\theta} r^{m-(n+1)} r^{n-1} d r+\int_{1}^{\infty} r^{\theta} r^{m-(n+1)} r^{-2 m} r^{n-1} d r\right) \\
& =n \omega_{n}\left(\left.\frac{r^{\theta+m-1}}{\theta+m-1}\right|_{0} ^{1}+\left.\frac{r^{\theta-m-1}}{\theta-m-1}\right|_{1} ^{\infty}\right)=\frac{2 n \omega_{n} m}{m^{2}-(1-\theta)^{2}}<\infty
\end{aligned}
$$

because $\theta+m-1>0(m \geq n \geq 1)$ and $\theta-m-1<0$ for all $\theta \in(0,1)$. Therefore, for $n \leq m<n+1$ and $|\alpha|=n+i, i=0,1$, there exists $\theta_{i} \in(0,1)$, such that

$$
\left\|(\mu \eta)^{\alpha} K_{\varepsilon}(\eta, \lambda)\right\| \leq C_{13} \mu^{n}|\mu \eta|^{\theta_{i}}
$$

for all $\lambda \in \sum_{\frac{\pi}{2}, R}$ and $\eta \in \mathbb{R}^{n}$.

We have shown that for $m>0$ and $|\alpha|=n, n+1$, there exists $\theta_{i} \in(0,1)$, $i=0,1$, such that

$$
\left\|(\mu \eta)^{\alpha} K_{\varepsilon}(\eta, \lambda)\right\| \leq C_{13} \mu^{n}|\mu \eta|^{\theta_{i}}
$$


for all $\lambda \in \sum_{\frac{\pi}{2}, R}$ and $\eta \in \mathbb{R}^{n}$. Therefore we have

$$
\begin{aligned}
|\mu \eta|^{n}\left\|K_{\varepsilon}(\eta, \lambda)\right\| & \leq n^{\frac{n}{2}} \sum_{|\alpha|=n}\left\|(\mu \eta)^{\alpha} K_{\varepsilon}(\eta, \lambda)\right\| \\
& \leq n^{\frac{n}{2}}\left(\sum_{|\alpha|=n} 1\right) C_{13} \mu^{n}|\mu \eta|^{\theta_{0}}
\end{aligned}
$$

because $|x|^{k} \leq n^{\frac{k}{2}} \sum_{|\alpha|=k}\left|x^{\alpha}\right|$ for all $x \in \mathbb{R}^{n}$. In the same way we obtain

$$
|\mu \eta|^{n+1}\left\|K_{\varepsilon}(\eta, \lambda)\right\| \leq n^{\frac{n+1}{2}}\left(\sum_{|\alpha|=n+1} 1\right) C_{13} \mu^{n}|\mu \eta|^{\theta_{1}} .
$$

From this two last inequalities assertion (i) follows with

$$
C^{*}:=n^{\frac{n+1}{2}}\left(\sum_{|\alpha|=n+1} 1\right) C_{13} .
$$

Proof of ii): Let $\varepsilon, \varepsilon^{\prime} \in(0,1), \xi, \eta \in \mathbb{R}^{n}$ and $\lambda \in \Sigma_{\frac{\pi}{2}, R}$. Then, analogous to the proof of Assertion i), it is obtained that

$$
\begin{aligned}
& (1+|\mu \eta|)|\mu \eta|^{n}\left\|K_{\varepsilon}(\eta, \lambda)-K_{\varepsilon^{\prime}}(\eta, \lambda)\right\|_{\mathcal{L}\left(E_{0}\right)} \\
& \leq \widetilde{c}_{n} \sum_{i=0,1} \sum_{|\alpha|=n+i} \mu^{n+m}|\mu \eta|^{\theta} \int_{\mathbb{R}^{n}}|\xi|^{\theta}\left\|\partial_{\xi}^{\alpha}\left[\left(\chi_{\varepsilon}(\mu \xi, \eta)-\chi_{\varepsilon^{\prime}}(\mu \xi, \eta)\right) b_{\lambda}(\mu \xi)\right]\right\|_{\mathcal{L}\left(E_{0}\right)} d \xi
\end{aligned}
$$

and

$$
\begin{aligned}
& |\xi|^{\theta}\left\|\partial_{\xi}^{\alpha}\left[\left(\chi_{\varepsilon}(\mu \xi, \eta)-\chi_{\varepsilon^{\prime}}(\mu \xi, \eta)\right) b_{\lambda}(\mu \xi)\right]\right\| \\
& \quad \leq \widetilde{C}_{n} \mu^{|\alpha|+n-m}|\xi|^{\theta}\langle\mu \xi\rangle^{m-|\alpha|}\langle\xi\rangle^{-2 m} \in L_{1}\left(\mathbb{R}_{\xi}^{n}\right) .
\end{aligned}
$$

From this, Lemma 3.12 and the dominated convergence Theorem it is obtained

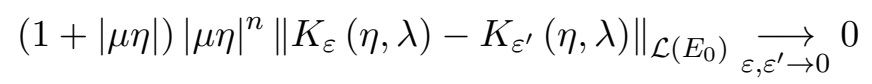

pointwise on $\mathbb{R}_{\eta}^{n} \times \Sigma_{\frac{\pi}{2}, R}$, and therefore there exists a strongly measurable function $K: \mathbb{R}_{\eta}^{n} \times \Sigma_{\frac{\pi}{2}, R} \rightarrow \mathcal{L}\left(E_{0}\right)$ with

$$
K_{\varepsilon}(\eta, \lambda) \underset{\varepsilon \rightarrow 0}{\longrightarrow} K(\eta, \lambda) \text { pointwise on } \mathbb{R}_{\eta}^{n} \times \Sigma_{\frac{\pi}{2}, R} .
$$

Furthermore the function $K$ satisfies, due to (51), the following:

$$
\|K(\eta, \lambda)\|_{\mathcal{L}\left(E_{0}\right)} \leq \frac{C^{*} \mu^{n}\left(|\mu \eta|^{\theta_{0}-n}+|\mu \eta|^{\theta_{1}-n}\right)}{1+|\mu \eta|} \in L_{1}\left(\mathbb{R}_{\eta}^{n}\right)
$$

for all $\lambda \in \Sigma_{\frac{\pi}{2}, R}$ and $a \in \mathcal{A}$. This shows ii).

Now we get from (49), the assertions i)-ii) and the dominated convergence theorem that for all $|\beta| \leq k, \lambda \in \Sigma_{\frac{\pi}{2}, R}$ and $u \in C_{b}^{\infty}\left(\mathbb{R}^{n}, E_{0}\right) \cap W_{p}^{k}\left(\mathbb{R}^{n}, E_{0}\right)$ :

$$
\begin{aligned}
\partial_{x}^{\beta}\left[\widetilde{b_{\lambda}(D)} u(x)\right] & =\int_{\mathbb{R}^{n}} K(\eta, \lambda)\left(\partial_{x}^{\beta} u\right)(x-\eta) \frac{d \eta}{|\lambda|(2 \pi)^{n}} \\
& =\frac{1}{|\lambda|(2 \pi)^{n}}\left[K(\cdot, \lambda) *\left(\partial_{x}^{\beta} u\right)\right](x) \in L_{p}\left(\mathbb{R}^{n}, E_{0}\right),
\end{aligned}
$$


since $\partial_{x}^{\beta} u \in L_{p}\left(\mathbb{R}^{n}, E_{0}\right)$. Then $\widetilde{b_{\lambda}(D)} u \in W_{p}^{k}\left(\mathbb{R}^{n}, E_{0}\right)$ and

$$
\left\|K(\cdot, \lambda) * \partial_{x}^{\beta} u\right\|_{L_{p}\left(\mathbb{R}^{n}, E_{0}\right)} \leq \frac{1}{(2 \pi)^{n}}\|K(\cdot, \lambda)\|_{L_{1}\left(\mathbb{R}_{\eta}^{n}, \mathcal{L}\left(E_{0}\right)\right)}\left\|\partial_{x}^{\beta} u\right\|_{L_{p}\left(\mathbb{R}^{n}, E_{0}\right)} .
$$

Thus, with $M_{1}:=\frac{2 \hat{C}}{(2 \pi)^{n}}$ it holds

$$
\begin{aligned}
& \left\|\left.\widetilde{b_{\lambda}(D)}\right|_{\mathcal{B}_{p, \infty}^{k}\left(\mathbb{R}^{n}, E_{0}\right)} u\right\|_{W_{p}^{k}\left(\mathbb{R}^{n}, E_{0}\right)} \\
& =\left(\sum_{|\alpha| \leq k}\left\|\partial_{x}^{\beta}\left(\widetilde{b_{\lambda}(D)} u\right)\right\|_{L_{p}\left(\mathbb{R}^{n}, E_{0}\right)}^{p}\right)^{\frac{1}{p}} \\
& \leq \frac{1}{(2 \pi)^{n}} \frac{1}{|\lambda|}\left(\sum_{|\beta| \leq k}\|K(\cdot, \lambda)\|_{L_{1}\left(\mathbb{R}_{\eta}^{n}, \mathcal{L}\left(E_{0}\right)\right)}^{p}\left\|\partial_{x}^{\beta} u\right\|_{L_{p}\left(\mathbb{R}^{n}, E_{0}\right)}^{p}\right)^{\frac{1}{p}} \\
& \leq \frac{M_{1}}{1+|\lambda|}\|u\|_{W_{p}^{k}\left(\mathbb{R}^{n}, E_{0}\right)} \quad(1 \leq p<\infty)
\end{aligned}
$$

for all $u \in C_{b}^{\infty}\left(\mathbb{R}^{n}, E_{0}\right) \cap W_{p}^{k}\left(\mathbb{R}^{n}, E_{0}\right), \lambda \in \Sigma_{\frac{\pi}{2}, R}$ and $a \in \mathcal{A}$. One proves the case $p=\infty$ in analogous form. For that, one must replace above $\sum_{|\beta| \leq k}$ by $\max _{|\beta| \leq k}$ and eliminate the exponent $p$.

In the following we prove the main theorem of this article.

Theorem 3.14. Let $m>0, k \in \mathbb{N}_{0}, \mathfrak{F}_{p}^{k}=W_{p}^{k}$ for $1 \leq p \leq \infty$ and $\mathcal{B}$ as in (40). We will assume that $m>k$ in case $p=\infty$. Moreover, let $\mathcal{A}:=$ $\left\{a_{\tau}: \tau \in \mathfrak{I}\right\} \subset S_{1,0}^{m, \rho_{n}}\left(\mathbb{R}^{n}, \mathcal{L}\left(E_{1}, E_{0}\right)\right)$ a bounded family of parabolic symbols with the same constants $\omega \geq 0$ and $\kappa>0, A(\tau):=\left.\widetilde{a_{\tau}(D)}\right|_{\mathcal{B}_{p, \infty}^{k+m}\left(\mathbb{R}^{n}, E_{1}\right)}$ for $\tau \in \mathfrak{I}$ and $A(\tau)_{W_{p}^{k}\left(\mathbb{R}^{n}, E_{0}\right)}$ the $W_{p}^{k}\left(\mathbb{R}^{n}, E_{0}\right)$-realization of $A(\tau)$. Then there are constants $M_{2}>0, M_{2}=C_{4}\left(M_{1}\right), 0<\vartheta<\pi / 2$ and $R>0$, such that $\Sigma_{\frac{\pi}{2}+\vartheta, R} \subset \rho\left(-A(t)_{W_{p}^{k}}\right)$ and

$$
\left\|\left(\lambda I+A(\tau)_{W_{p}^{k}\left(\mathbb{R}^{n}, E_{0}\right)}\right)^{-1}\right\|_{\mathcal{L}\left(W_{p}^{k}\left(\mathbb{R}^{n}, E_{0}\right)\right)} \leq \frac{M_{2}}{1+|\lambda|}
$$

for all $\lambda \in \Sigma_{\frac{\pi}{2}+\vartheta, R}$ and $\tau \in \mathfrak{I}$, with $M_{1}$ the constant given in Proposition 3.13 and $C_{4}\left(M_{1}\right)$ defined like in Proposition 3.3. Therefore

$$
-A(\tau)_{W_{p}^{k}}: D\left(A(\tau)_{W_{p}^{k}}\right) \longrightarrow W_{p}^{k}\left(\mathbb{R}^{n}, E_{0}\right)
$$

generates an analytic semigroup on $W_{p}^{k}\left(\mathbb{R}^{n}, E_{0}\right)$. Moreover

$$
\begin{gathered}
\stackrel{\circ}{B}_{p, 1}^{k+m}\left(\mathbb{R}^{n}, E_{1}\right) \stackrel{d}{\hookrightarrow} D\left(A(\tau)_{W_{p}^{k}\left(\mathbb{R}^{n}, E_{0}\right)}\right) \stackrel{d}{\hookrightarrow} \stackrel{\circ}{B}_{p, \infty}^{k+m}\left(\mathbb{R}^{n}, E_{1}\right), \quad 1 \leq p<\infty \\
B_{\infty, 1}^{k+m}\left(\mathbb{R}^{n}, E_{1}\right) \hookrightarrow D\left(A(\tau)_{W_{\infty}^{k}\left(\mathbb{R}^{n}, E_{0}\right)}\right) \hookrightarrow B_{\infty, \infty}^{k+m}\left(\mathbb{R}^{n}, E_{1}\right) .
\end{gathered}
$$


Proof. From (43) we know that

$$
\left(\lambda I+A(\tau)_{W_{p}^{k}\left(\mathbb{R}^{n}, E_{0}\right)}\right)^{-1}=\left.\left(\lambda I+\left.\widetilde{a_{\tau}(D)}\right|_{\mathcal{B}_{p, \infty}^{k+m}\left(\mathbb{R}^{n}, E_{1}\right)}\right)^{-1}\right|_{W_{p}^{k}\left(\mathbb{R}^{n}, E_{0}\right)} .
$$

Moreover it follows from Proposition 3.5 the existence of a constant $R_{1} \geq 0$ such that

$$
\left.\widetilde{b_{\lambda}(D)}\right|_{B_{p, q}^{k}\left(\mathbb{R}^{n}, E_{0}\right)}=\left(\lambda I+\left.\widetilde{a_{\tau}(D)}\right|_{B_{p, q}^{k+m}\left(\mathbb{R}^{n}, E_{1}\right)}\right)^{-1}
$$

for all $\lambda \in \Sigma_{\frac{\pi}{2}, R_{1}}, \tau \in \mathfrak{I}, p, q \in[1, \infty]$. From this it follows immediately

$$
\left(\lambda I+A(\tau)_{W_{\infty}^{k}\left(\mathbb{R}^{n}, E_{0}\right)}\right)^{-1}=\left.\widetilde{b_{\lambda}(D)}\right|_{B_{\infty, \infty}^{k}\left(\mathbb{R}^{n}, E_{0}\right)} \text { on } W_{\infty}^{k}\left(\mathbb{R}^{n}, E_{0}\right) .
$$

On the other hand, since the maps

$$
\begin{array}{cccc}
\lambda I+\left.\widetilde{a_{\tau}(D)}\right|_{B_{p, \infty}^{k+m}\left(\mathbb{R}^{n}, E_{1}\right)}: & B_{p, \infty}^{k+m}\left(\mathbb{R}^{n}, E_{1}\right) & \longrightarrow & B_{p, \infty}^{k}\left(\mathbb{R}^{n}, E_{0}\right) \\
\lambda I+\left.\widetilde{a_{\tau}(D)}\right|_{\dot{B}_{p, \infty}^{k+m}\left(\mathbb{R}^{n}, E_{1}\right)}: & \stackrel{\circ}{B}_{p, \infty}^{k+m}\left(\mathbb{R}^{n}, E_{1}\right) & \longrightarrow & \stackrel{\circ}{B}_{p, \infty}^{k}\left(\mathbb{R}^{n}, E_{0}\right)
\end{array}
$$

are bijective, it holds that

$$
\begin{aligned}
\left(\lambda I+\left.\widetilde{a_{\tau}(D)}\right|_{\hat{B}_{p, \infty}^{k+m}\left(\mathbb{R}^{n}, E_{1}\right)}\right. & \\
= & \left.\left(\lambda I+\left.\widetilde{a_{\tau}(D)}\right|_{B_{p, \infty}^{k+m}\left(\mathbb{R}^{n}, E_{1}\right)}\right)^{-1}\right|_{B_{p, \infty}^{k}\left(\mathbb{R}^{n}, E_{0}\right)} .
\end{aligned}
$$

Furthermore one obtains from $W_{p}^{k}\left(\mathbb{R}^{n}, E_{0}\right) \hookrightarrow \stackrel{\circ}{B_{p, \infty}^{k}}\left(\mathbb{R}^{n}, E_{0}\right), 1 \leq p<\infty$, that

$$
\begin{aligned}
&\left(\lambda I+A(\tau)_{W_{p}^{k}\left(\mathbb{R}^{n}, E_{0}\right)}\right)^{-1}\left.\stackrel{(60)}{=}\left(\lambda I+\left.\widetilde{a_{\tau}(D)}\right|_{B_{p, \infty}^{k+m}\left(\mathbb{R}^{n}, E_{1}\right)}\right)^{-1}\right|_{W_{p}^{k}\left(\mathbb{R}^{n}, E_{0}\right)} \\
&\left.\stackrel{(58)}{=} \widetilde{b_{\lambda}(D)}\right|_{W_{p}^{k}\left(\mathbb{R}^{n}, E_{0}\right)}
\end{aligned}
$$

Then, according to Proposition 3.13, there exist a constant $R_{2} \geq R_{1}$ such that for all $\lambda \in \Sigma_{\frac{\pi}{2}, R_{2}}$ and $\tau \in \mathfrak{I}$ :

$$
\begin{aligned}
& \left\|\left(\lambda I+A(\tau)_{W_{p}^{k}\left(\mathbb{R}^{n}, E_{0}\right)}\right)^{-1} u\right\|_{W_{p}^{k}\left(\mathbb{R}^{n}, E_{0}\right)} \leq \frac{M_{1}}{1+|\lambda|}\|u\|_{W_{p}^{k}\left(\mathbb{R}^{n}, E_{0}\right)} \\
& \forall u \in \mathcal{S}\left(\mathbb{R}^{n}, E_{0}\right), 1 \leq p<\infty, \\
& \left\|\left(\lambda I+A(\tau)_{W_{\infty}^{k}\left(\mathbb{R}^{n}, E_{0}\right)}\right)^{-1} u\right\|_{W_{\infty}^{k}\left(\mathbb{R}^{n}, E_{0}\right)} \leq \frac{M_{1}}{1+|\lambda|}\|u\|_{W_{\infty}^{k}\left(\mathbb{R}^{n}, E_{0}\right)} \\
& \forall u \in C_{b}^{\infty}\left(\mathbb{R}^{n}, E_{0}\right) .
\end{aligned}
$$


If $1 \leq p<\infty, \mathcal{S}\left(\mathbb{R}^{n}, E_{0}\right) \stackrel{d}{\hookrightarrow} W_{p}^{k}\left(\mathbb{R}^{n}, E_{0}\right)$ and therefore

$$
\left\|\left(\lambda I+A(\tau)_{W_{p}^{k}\left(\mathbb{R}^{n}, E_{0}\right)}\right)^{-1}\right\|_{\mathcal{L}\left(W_{p}^{k}\left(\mathbb{R}^{n}, E_{0}\right)\right)} \leq \frac{M_{1}}{1+|\lambda|}, \quad \text { if } 1 \leq p<\infty .
$$

Now, we prove the inequality (63) for all $u \in W_{\infty}^{k}\left(\mathbb{R}^{n}, E_{0}\right)$ under the hypothesis that in this case $m>k$. Let $m>k$, then there exists some $r_{1} \in \mathbb{R}$ (e.g. $r_{1}=\frac{k-m}{2}$ ) with $r_{1}<k<r_{1}+m$. Now, we choose a $R_{3} \geq R_{2}$ such that it holds (57), (63), for all $\lambda \in \Sigma_{\frac{\pi}{2}, R_{3}} \mathrm{y} \tau \in \mathfrak{I}$ and such that the maps

$$
\begin{array}{cccc}
\lambda I+\left.\widetilde{a_{\tau}(D)}\right|_{B_{\infty, 1}^{r_{1}+m}\left(\mathbb{R}^{n}, E_{1}\right)}: & B_{\infty, 1}^{r_{1}+m}\left(\mathbb{R}^{n}, E_{1}\right) & \longrightarrow & B_{\infty, 1}^{r_{1}}\left(\mathbb{R}^{n}, E_{0}\right) \\
\lambda I+\left.\widetilde{a_{\tau}(D)}\right|_{B_{\infty, \infty}^{k+m}\left(\mathbb{R}^{n}, E_{1}\right)}: & B_{\infty, \infty}^{k+m}\left(\mathbb{R}^{n}, E_{1}\right) & \longrightarrow & B_{\infty, \infty}^{k}\left(\mathbb{R}^{n}, E_{0}\right)
\end{array}
$$

are bijective and continuous (this is possible due to Corollary 3.2 and Proposition 3.5). Then

$$
\left(\lambda I+\left.\widetilde{a_{\tau}(D)}\right|_{B_{\infty, \infty}^{k+m}\left(\mathbb{R}^{n}, E_{1}\right)}\right)^{-1}=\left.\left(\lambda I+\left.\widetilde{a_{\tau}(D)}\right|_{B_{\infty, 1}^{r_{1}+m}\left(\mathbb{R}^{n}, E_{1}\right)}\right)^{-1}\right|_{B_{\infty, \infty}^{k}\left(\mathbb{R}^{n}, E_{0}\right)} .
$$

Now, let $u \in W_{\infty}^{k}\left(\mathbb{R}^{n}, E_{0}\right)$ and $\psi \in \mathcal{S}\left(\mathbb{R}^{n}\right)$ with $\psi \equiv 1$ on $\overline{B_{1}(0)}, 0 \leq \psi \leq 1$, $\psi \equiv 0$ on $\mathbb{R}^{n} \backslash B_{2}(0)$ and $\|\check{\psi}\|_{L_{1}\left(\mathbb{R}^{n}\right)} \leq 1$. Moreover, let $\psi_{l}(\xi):=\psi\left(2^{-l} \xi\right)$ for $\xi \in \mathbb{R}^{n}, l \in \mathbb{N}, r_{2} \in \mathbb{R}$ with $r_{1}<r_{2}<k$ and $r:=r_{2}-r_{1}>0$. It is clear that $\psi_{l} \in S_{1,0}^{r, \rho_{n}}\left(\mathbb{R}^{n}\right)$ and $u_{l}:=\psi_{l}(D) u \in C_{b}^{\infty}\left(\mathbb{R}^{n}, E_{0}\right)$. From this, (57), (65) and (37) it follows that there are constants $c_{i}, i=1,2,3$, such that for all $\lambda \in \Sigma_{\frac{\pi}{2}, R_{3}}$ and $\tau \in \mathfrak{I}:$

$$
\begin{aligned}
& \left\|\left(\lambda I+A(\tau)_{W_{\infty}^{k}\left(\mathbb{R}^{n}, E_{0}\right)}\right)^{-1}\left(u-u_{l}\right)\right\|_{W_{\infty}^{k}\left(\mathbb{R}^{n}, E_{0}\right)} \\
& =\left\|\left(\lambda I+\left.\widetilde{a_{\tau}(D)}\right|_{B_{\infty, \infty}^{k+m}\left(\mathbb{R}^{n}, E_{1}\right)}\right)^{-1}\left(u-u_{l}\right)\right\|_{W_{\infty}^{k}\left(\mathbb{R}^{n}, E_{0}\right)} \\
& \leq c_{1}\left\|\left(\lambda I+\left.\widetilde{a_{\tau}(D)}\right|_{B_{\infty, 1}^{r_{1}+m}\left(\mathbb{R}^{n}, E_{1}\right)}\right)^{-1}\left(u-u_{l}\right)\right\|_{B_{\infty, 1}^{r_{1}+m}\left(\mathbb{R}^{n}, E_{1}\right)} \\
& \leq c_{2}\left\|u-u_{l}\right\|_{B_{\infty, 1}^{r_{1}}\left(\mathbb{R}^{n}, E_{0}\right)} \\
& =c_{2}\left\|\left(I-\psi_{l}(D)\right) u\right\|_{B_{\infty}^{r_{1}, 1}\left(\mathbb{R}^{n}, E_{0}\right)} \\
& \leq c_{3}\left\|1-\psi_{l}\right\|_{S_{1,0}^{r, n+1}\left(\mathbb{R}^{n}\right)}\|u\|_{B_{\infty, 1}^{r_{2}}\left(\mathbb{R}^{n}, E_{0}\right)} \\
& \longrightarrow 0, \text { when } l \rightarrow \infty .
\end{aligned}
$$

Thus

$$
\left(\lambda I+A(\tau)_{W_{\infty}^{k}\left(\mathbb{R}^{n}, E_{0}\right)}\right)^{-1} u_{l} \stackrel{l \rightarrow \infty}{\longrightarrow}\left(\lambda I+A(\tau)_{W_{\infty}^{k}\left(\mathbb{R}^{n}, E_{0}\right)}\right)^{-1} u \text { in } W_{\infty}^{k}\left(\mathbb{R}^{n}, E_{0}\right)
$$

for all $\tau \in \mathfrak{I}$. Since

$$
\left\|\left(\psi_{l}(D)\right) u\right\|_{W_{\infty}^{k}\left(\mathbb{R}^{n}, E_{0}\right)}=(2 \pi)^{-\frac{n}{2}}\left\|\check{\psi}_{l} * u\right\|_{W_{\infty}^{k}\left(\mathbb{R}^{n}, E_{0}\right)}
$$


PSEUDODIFFERENTIAL OPERATORS ON VECTOR-VALUED SOBOLEV SPACES 27

$$
\begin{aligned}
& =(2 \pi)^{-\frac{n}{2}} \max _{|\alpha| \leq k}\left\|\check{\psi}_{l} * \partial_{x}^{\alpha} u\right\|_{L_{\infty}\left(\mathbb{R}^{n}, E_{0}\right)} \\
& \leq\left\|\check{\psi}_{l}\right\|_{L_{1}\left(\mathbb{R}^{n}\right)} \max _{|\alpha| \leq k}\left\|\partial_{x}^{\alpha} u\right\|_{L_{\infty}\left(\mathbb{R}^{n}, E_{0}\right)} \\
& \leq\|u\|_{W_{\infty}^{k}\left(\mathbb{R}^{n}, E_{0}\right)},
\end{aligned}
$$

we get from (63) that

$$
\begin{aligned}
\left\|\left(\lambda I+A(\tau)_{W_{\infty}^{k}\left(\mathbb{R}^{n}, E_{0}\right)}\right)^{-1} u_{l}\right\|_{W_{\infty}^{k}\left(\mathbb{R}^{n}, E_{0}\right)} & \leq \frac{M_{1}}{1+|\lambda|}\left\|u_{l}\right\|_{W_{\infty}^{k}\left(\mathbb{R}^{n}, E_{0}\right)} \\
& \leq \frac{M_{1}}{1+|\lambda|}\|u\|_{W_{\infty}^{k}\left(\mathbb{R}^{n}, E_{0}\right)}
\end{aligned}
$$

for all $l \in \mathbb{N}, \lambda \in \Sigma_{\frac{\pi}{2}, R_{3}}$ and $\tau \in \mathfrak{I}$. Therefore

$$
\begin{aligned}
\left\|\left(\lambda I+A(\tau)_{W_{\infty}^{k}\left(\mathbb{R}^{n}, E_{0}\right)}\right)^{-1} u\right\|_{W_{\infty}^{k}\left(\mathbb{R}^{n}, E_{0}\right)} & \leq \frac{M_{1}}{1+|\lambda|}\|u\|_{W_{\infty}^{k}\left(\mathbb{R}^{n}, E_{0}\right)} \\
& \text { for all } u \in W_{\infty}^{k}\left(\mathbb{R}^{n}, E_{0}\right),
\end{aligned}
$$

$\lambda \in \Sigma_{\frac{\pi}{2}, R_{3}}$ and $\tau \in \mathfrak{I}$. Then (54) follows from Proposition 3.3. The assertions (55) and (56) follow from Corollary 3.2 and from equality $\stackrel{\circ}{B}_{p, \infty}^{k}\left(\mathbb{R}^{n}, E_{1}\right)=b_{p, \infty}^{k}\left(\mathbb{R}^{n}, E_{1}\right) \quad$ for $1 \leq p<\infty$. In fact, from Definition 3.7 with

$$
\begin{array}{r}
A(\tau): D(A(\tau)) \subset b_{p, \infty}^{k}\left(\mathbb{R}^{n}, E_{0}\right)=: \mathbb{E}_{0} \longrightarrow \mathbb{E}_{0} \text { and } \\
A(\tau)_{W_{p}^{k}}: D\left(A(\tau)_{W_{p}^{k}}\right) \subset W_{p}^{k}\left(\mathbb{R}^{n}, E_{0}\right)=: \mathbb{E}_{1} \longrightarrow \mathbb{E}_{1},
\end{array}
$$

$p<\infty$, we get that

$$
D\left(A(\tau)_{W_{p}^{k}}\right)=\left\{u \in b_{p, \infty}^{k+m}\left(\mathbb{R}^{n}, E_{1}\right):\left.\widetilde{a_{\tau}(D)}\right|_{b_{p, \infty}^{k+m}\left(\mathbb{R}^{n}, E_{1}\right)} u \in W_{p}^{k}\left(\mathbb{R}^{n}, E_{0}\right)\right\}
$$

and therefore ${ }^{10}$

$$
B_{p, 1}^{k+m}\left(\mathbb{R}^{n}, E_{1}\right) \hookrightarrow D\left(A(\tau)_{W_{p}^{k}\left(\mathbb{R}^{n}, E_{0}\right)}\right) \hookrightarrow b_{p, \infty}^{k+m}\left(\mathbb{R}^{n}, E_{1}\right), \quad 1 \leq p<\infty .
$$

Let $u \in b_{p, \infty}^{k+m}\left(\mathbb{R}^{n}, E_{1}\right)$. Then there is (due to definition of these spaces) a sequence $\left(u_{l}\right)_{l \in \mathbb{N}} \subset B_{p, \infty}^{k+m+1}\left(\mathbb{R}^{n}, E_{1}\right)$ with $u_{l} \rightarrow u$ in $B_{p, \infty}^{k+m}\left(\mathbb{R}^{n}, E_{1}\right)$. Furthermore, it follows from (28) and $B_{p, \infty}^{k+m+1}\left(\mathbb{R}^{n}, E_{1}\right) \hookrightarrow b_{p, \infty}^{k+m}\left(\mathbb{R}^{n}, E_{1}\right)$ that

$$
\left.\widetilde{a_{\tau}(D)}\right|_{b_{p, \infty}^{k+m}\left(\mathbb{R}^{n}, E_{1}\right)} u_{l} \in B_{p, \infty}^{k+1}\left(\mathbb{R}^{n}, E_{1}\right) \hookrightarrow W_{p}^{k}\left(\mathbb{R}^{n}, E_{0}\right) .
$$

So, one concludes that $D\left(A(\tau)_{W_{p}^{k}}\right) \stackrel{d}{\hookrightarrow} b_{p, \infty}^{k+m}\left(\mathbb{R}^{n}, E_{1}\right)$. Now, let $u \in D\left(A(\tau)_{W_{p}^{k}}\right)$. From definition of $D\left(A(\tau)_{W_{p}^{k}}\right)$ it is obtained that

$$
v:=\left(\lambda I+\left.\widetilde{a_{\tau}(D)}\right|_{b_{p, \infty}^{k+m}\left(\mathbb{R}^{n}, E_{1}\right)}\right) u \in W_{p}^{k}\left(\mathbb{R}^{n}, E_{0}\right)
$$

\footnotetext{
${ }^{10} D\left(A(t)_{W_{p}^{k}\left(\mathbb{R}^{n}, E_{0}\right)}\right)$ endowed with the graph norm (39).
} 
for some $\lambda \in \rho\left(-A(\tau)_{W_{p}^{k}}\right)$. Since $B_{p, 1}^{k}\left(\mathbb{R}^{n}, E_{0}\right) \stackrel{d}{\hookrightarrow} W_{p}^{k}\left(\mathbb{R}^{n}, E_{0}\right)$ for $1 \leq p<$ $\infty$, there exists a sequence $\left(v_{l}\right)_{l \in \mathbb{N}} \subset B_{p, 1}^{k}\left(\mathbb{R}^{n}, E_{0}\right)$ with

$$
v_{l} \longrightarrow v \text { in } W_{p}^{k}\left(\mathbb{R}^{n}, E_{0}\right) .
$$

We know that the application

$$
\begin{array}{r}
\left(\lambda I+A(\tau)_{W_{p}^{k}}\right)^{-1}=\left.\left(\lambda I+\left.\widetilde{a_{\tau}(D)}\right|_{b_{p, \infty}^{k+m}\left(\mathbb{R}^{n}, E_{1}\right)}\right)^{-1}\right|_{W_{p}^{k}\left(\mathbb{R}^{n}, E_{0}\right)} \\
: W_{p}^{k}\left(\mathbb{R}^{n}, E_{0}\right) \longrightarrow W_{p}^{k}\left(\mathbb{R}^{n}, E_{0}\right)
\end{array}
$$

is continuous. Therefore

$$
u_{l}:=\left(\lambda I+\left.\widetilde{a_{\tau}(D)}\right|_{b_{p, \infty}^{k+m}\left(\mathbb{R}^{n}, E_{1}\right)}\right)^{-1} v_{l} \longrightarrow u \text { in } W_{p}^{k}\left(\mathbb{R}^{n}, E_{0}\right) .
$$

On the other hand one deduces from

$$
B_{p, 1}^{k+m}\left(\mathbb{R}^{n}, E_{1}\right)=b_{p, 1}^{k+m}\left(\mathbb{R}^{n}, E_{1}\right) \hookrightarrow b_{p, \infty}^{k+m}\left(\mathbb{R}^{n}, E_{1}\right)
$$

(see Lemma 2.1e) and (21)) that

$$
\begin{aligned}
u_{l} & =\left(\lambda I+\left.\widetilde{a_{\tau}(D)}\right|_{b_{p, \infty}^{k+m}\left(\mathbb{R}^{n}, E_{1}\right)}\right)^{-1} v_{l} \\
& =\left(\lambda I+\left.\widetilde{a_{\tau}(D)}\right|_{B_{p, 1}^{k+m}\left(\mathbb{R}^{n}, E_{1}\right)}\right)^{-1} v_{l} \in B_{p, 1}^{k+m}\left(\mathbb{R}^{n}, E_{1}\right)
\end{aligned}
$$

for all $l \in \mathbb{N}$. Then, according to (68)-(70), there exists a sequence $\left(u_{l}\right)_{l \in \mathbb{N}} \subset$ $B_{p, 1}^{k+m}\left(\mathbb{R}^{n}, E_{1}\right)$ such that

$$
\begin{aligned}
\left\|u-u_{l}\right\|_{D\left(A_{W_{p}^{k}}\right)} & =\left\|u-u_{l}\right\|_{W_{p}^{k}}+\left\|\left.\widetilde{a_{\tau}(D)}\right|_{b_{p, \infty}^{k+m}\left(\mathbb{R}^{n}, E_{1}\right)}\left(u-u_{l}\right)\right\|_{W_{p}^{k}} \\
& =\left\|u-u_{l}\right\|_{W_{p}^{k}}+\left\|v-\lambda u-v_{l}+\lambda u_{l}\right\|_{W_{p}^{k}} \\
& \leq(1+|\lambda|)\left\|u-u_{l}\right\|_{W_{p}^{k}}+\left\|v-v_{l}\right\|_{W_{p}^{k}} \longrightarrow 0, \text { if } l \rightarrow \infty .
\end{aligned}
$$

Consequently $B_{p, 1}^{k+m}\left(\mathbb{R}^{n}, E_{1}\right)$ is dense in $D\left(A(\tau)_{W_{p}^{k}}\right)$ for $1 \leq p<\infty$ and with this (55) is proven. Analogous one shows (56).

Corollary 3.15. Let conditions as in Theorem 3.14 for the case $p=\infty$, $m>k$, but now $\mathfrak{F}_{\infty}^{k} \in\left\{B U C^{k}, C_{0}^{k}, C_{b}^{k}\right\}$ and let $\mathcal{B}$ be as in (40). Then

a) The operator

$$
-A(\tau)_{\mathfrak{F}_{\infty}^{k}}: D\left(A(\tau)_{\mathfrak{F}_{\infty}^{k}}\right) \longrightarrow \mathfrak{F}_{\infty}^{k}\left(\mathbb{R}^{n}, E_{0}\right)
$$

generates an analytic semigroup on $\mathfrak{F}_{\infty}^{k}\left(\mathbb{R}^{n}, E_{0}\right)$ for all $\tau \in \mathfrak{I}$. Exactly, there exist constants $\vartheta \in] 0, \frac{\pi}{2}\left[\right.$ and $R>0$ such that $\rho\left(-A(\tau)_{\mathfrak{F}_{\infty}^{k}}\right) \subset$ $\Sigma_{\frac{\pi}{2}+\vartheta, R}$ and

$$
\left\|\left(\lambda I+A(\tau)_{\mathfrak{F}_{\infty}^{k}}\right)^{-1}\right\|_{\mathcal{L}\left(\mathfrak{F}_{\infty}^{k}\right)} \leq \frac{M_{2}}{1+|\lambda|}
$$

for all $\lambda \in \Sigma_{\frac{\pi}{2}+\vartheta, R}$ and $\tau \in \mathfrak{I}$. 
b)

$$
\begin{aligned}
& b_{\infty, 1}^{k+m}\left(\mathbb{R}^{n}, E_{1}\right) \stackrel{d}{\hookrightarrow} D\left(A(\tau)_{B U C^{k}\left(\mathbb{R}^{n}, E_{0}\right)}\right) \stackrel{d}{\hookrightarrow} b_{\infty, \infty}^{k+m}\left(\mathbb{R}^{n}, E_{1}\right), \\
& \stackrel{\circ}{B}_{\infty, 1}^{k+m}\left(\mathbb{R}^{n}, E_{1}\right) \stackrel{d}{\hookrightarrow} D\left(A(\tau)_{C_{0}^{k}\left(\mathbb{R}^{n}, E_{0}\right)}\right) \stackrel{d}{\hookrightarrow} \stackrel{\circ}{B}_{\infty, \infty}^{k+m}\left(\mathbb{R}^{n}, E_{1}\right), \\
& B_{\infty, 1}^{k+m}\left(\mathbb{R}^{n}, E_{1}\right) \hookrightarrow D\left(A(\tau)_{C_{b}^{k}\left(\mathbb{R}^{n}, E_{0}\right)}\right) \hookrightarrow B_{\infty, \infty}^{k+m}\left(\mathbb{R}^{n}, E_{1}\right) .
\end{aligned}
$$

Proof. a) From $\mathcal{B}_{\infty, \infty}^{k+m}\left(\mathbb{R}^{n}, E_{0}\right) \cap \mathfrak{F}_{\infty}^{k}\left(\mathbb{R}^{n}, E_{0}\right)=\mathcal{B}_{\infty, \infty}^{k+m}\left(\mathbb{R}^{n}, E_{0}\right)$ and from definition of the realization of a operator one obtains that

$$
\begin{aligned}
D\left(A(\tau)_{\mathfrak{F}_{\infty}^{k}}\right) & =\left\{u \in \mathcal{B}_{\infty, \infty}^{k+m}\left(\mathbb{R}^{n}, E_{0}\right):\left.\widetilde{a_{\tau}(D)}\right|_{\mathcal{B}_{\infty, \infty}^{k+m}\left(\mathbb{R}^{n}, E_{0}\right)} u \in \mathfrak{F}_{\infty}^{k}\left(\mathbb{R}^{n}, E_{0}\right)\right\}, \\
A(\tau)_{\mathfrak{F}_{\infty}^{k}} u & =\left.\widetilde{a_{\tau}(D)}\right|_{\mathcal{B}_{\infty, \infty}^{k+m}\left(\mathbb{R}^{n}, E_{0}\right)} u \quad \text { for } u \in D\left(A(\tau)_{\mathfrak{F}_{\infty}^{k}}\right)
\end{aligned}
$$

and

$$
\begin{aligned}
D\left(A(\tau)_{W_{\infty}^{k}}\right) & =\left\{u \in B_{\infty, \infty}^{k+m}\left(\mathbb{R}^{n}, E_{0}\right):\left.\widetilde{a_{\tau}(D)}\right|_{B_{\infty, \infty}^{k+m}\left(\mathbb{R}^{n}, E_{0}\right)} u \in W_{\infty}^{k}\left(\mathbb{R}^{n}, E_{0}\right)\right\}, \\
A(\tau)_{W_{\infty}^{k}} u & =\left.\widetilde{a_{\tau}(D)}\right|_{B_{\infty, \infty}^{k+m}\left(\mathbb{R}^{n}, E_{0}\right)} u \text { for all } u \in D\left(A(\tau)_{W_{\infty}^{k}}\right) .
\end{aligned}
$$

Because of $\mathcal{B}_{\infty, \infty}^{k+m}\left(\mathbb{R}^{n}, E_{0}\right) \hookrightarrow B_{\infty, \infty}^{k+m}\left(\mathbb{R}^{n}, E_{0}\right)($ see $(19))$ and $\mathfrak{F}_{\infty}^{k}\left(\mathbb{R}^{n}, E_{0}\right) \hookrightarrow$ $W_{\infty}^{k}\left(\mathbb{R}^{n}, E_{0}\right)$, we have

$D\left(A(\tau)_{\mathfrak{F}_{\infty}^{k}}\right) \subset D\left(A(\tau)_{W_{\infty}^{k}}\right)$ and $A(\tau)_{\mathfrak{F}_{\infty}^{k}}=A(\tau)_{W_{\infty}^{k}}$ on $D\left(A(\tau)_{\mathfrak{F}_{\infty}^{k}}\right)$.

By Proposition 3.8 there exists a constant $R>0$ such that for all $\lambda \in \sum_{\frac{\pi}{2}, R}$, $\tau \in \mathfrak{I}$ the maps

$$
\begin{array}{cccc}
\lambda I+A(\tau)_{W_{\infty}^{k}}: & D\left(A(\tau)_{W_{\infty}^{k}}\right) & \longrightarrow & W_{\infty}^{k}\left(\mathbb{R}^{n}, E_{0}\right) \\
\cup I+A(\tau)_{\mathfrak{F}_{\infty}^{k}}: & D\left(A(\tau)_{\mathfrak{F}_{\infty}^{k}}\right) & \longrightarrow & \mathfrak{F}_{\infty}^{k}\left(\mathbb{R}^{n}, E_{0}\right)
\end{array}
$$

are bijective, and so we infer that

$$
\left(\lambda I+A(\tau)_{\mathfrak{F}_{\infty}^{k}}\right)^{-1}=\left(\lambda I+A(\tau)_{W_{\infty}^{k}}\right)^{-1} \text { on } \mathfrak{F}_{\infty}^{k}\left(\mathbb{R}^{n}, E_{0}\right) .
$$

From this, from Theorem 3.14 and definitions of the norms $\|\cdot\|_{W_{\infty}^{k}} \mathrm{y}\|\cdot\|_{\mathfrak{F}_{\infty}^{k}}$ we conclude the assertion (71). $b$ ) The proofs of the continuous immersions in (72) are similar to the proofs of (55) and (56).

Remark 3.16. Let $m \in \mathbb{R}$ and $\rho \in \mathbb{N}_{0}$. We define $S^{m, \rho}\left(\mathbb{R}^{n}, E\right)$ as the space of all functions $a \in S_{1,0}^{m, \rho}\left(\mathbb{R}^{n}, E\right)$, which have a function $a^{0} \in S_{1,0}^{m, \rho}\left(\mathbb{R}^{n}, E\right)$, so that

$$
\begin{aligned}
& a-a^{0} \in S_{1,0}^{m-1, \rho}\left(\mathbb{R}^{n}, E\right) \text { and } \\
& a^{0}(t \xi)=t^{m} a^{0}(\xi) \text { for all } t>0 \text { and } \xi \in \mathbb{R}^{n} \backslash\{0\} .
\end{aligned}
$$

The symbol $a^{0}$ is called the main part of a and we regard $S^{m, \rho}\left(\mathbb{R}^{n}, E\right)$ with the norm

$$
\|a\|_{S^{m, \rho}\left(\mathbb{R}^{n}, E\right)}:=\left\|a^{0}\right\|_{S^{m, \rho}\left(\mathbb{R}^{n}, E\right)}+\left\|a-a^{0}\right\|_{S^{m-1, \rho}\left(\mathbb{R}^{n}, E\right)} .
$$


Furthermore, we say that $a \in S^{m, \rho} \mathbb{R}^{n}, \mathcal{L}\left(E_{1}, E_{0}\right), m \in \mathbb{R}^{+}, \rho \in \mathbb{N}_{0}$, is parabolic in $S^{m, \rho}\left(\mathbb{R}^{n}, \mathcal{L}\left(E_{1}, E_{0}\right)\right)$ if, and only if, a $a^{0}$ is parabolic as explained in Definition 3.4. One can prove that:

i): If $a_{\tau} \in S^{m, \rho_{n}} \mathbb{R}^{n}, \mathcal{L}\left(E_{1}, E_{0}\right), \tau \in \mathfrak{I}$, then

$$
D\left(A^{0}(\tau)_{\mathfrak{F}_{p}^{k}}\right)=D\left(A(\tau)_{\mathfrak{F}_{p}^{k}}\right), \quad \tau \in \mathfrak{I},
$$

where $A(\tau)_{\mathfrak{F}_{p}^{k}}$ and $A^{0}(\tau)_{\mathfrak{F}_{p}^{k}}$ are defined as in Theorem 3.14.

ii): If a is a parabolic symbol in $S^{m, \rho_{n}}\left(\mathbb{R}^{n}, \mathcal{L}\left(E_{1}, E_{0}\right)\right)$, then a is parabolic in $S_{1,0}^{m, \rho_{n}}\left(\mathbb{R}^{n}, \mathcal{L}\left(E_{1}, E_{0}\right)\right)$.

\section{Aplication}

4.1. An abstract linear Cauchy problem. Let $T>0, J:=[0, T]$ a closed interval in $\mathbb{R}$ and $t \in J$. In the following $t$ in $a(t, \cdot) \in S_{1,0}^{m, \rho_{n}}\left(\mathbb{R}^{n}, \mathcal{L}(E)\right)$ and in $\widetilde{a(t, D)}$ denotes only a parameter. Moreover we will consider in this section a family $\mathcal{A}:=\{a(t, \cdot): t \in J\} \subset S_{1,0}^{m, \rho_{n}}\left(\mathbb{R}^{n}, \mathcal{L}(E)\right)$ of parabolic symbols with the same constants $\omega \geq 0$ and $\kappa>0$ such that

$$
J \ni t \longmapsto a(t, \cdot) \in S_{1,0}^{m, \rho_{n}}\left(\mathbb{R}^{n}, \mathcal{L}(E)\right)
$$

is a Hölder continuous function relative to the topology in the space of the symbols, and we will use the results of the previous sections to study the existence and uniqueness of solutions for the Cauchy problem

$$
\left\{\begin{aligned}
\partial_{t} u+A(t)_{\mathfrak{F}_{p}^{k}} u & =f(t), \quad t \in J \backslash\{0\}, \\
u(0) & =u_{0},
\end{aligned}\right.
$$

in $\mathfrak{F}_{p}^{k}\left(\mathbb{R}^{n}, E_{0}\right)$. There, $A(t)=\widetilde{a(t, D)}, t \in J$, and $f:[0, T] \rightarrow \mathfrak{F}_{p}^{k}\left(\mathbb{R}^{n}, E\right)$ is a given Hölder continuous function. A function

$$
\left.\left.\left.\left.u \in C^{1}(] 0, T\right], E\right) \cap C(] 0, T\right], D(A(t))\right) \cap C([0, T], E)
$$

is called classical solution of $(73)$ in $[0, T]$, if $u^{\prime}(t)+A(t)_{\mathfrak{F}_{p}^{k}} u(t)=f(t)$ for all $t \in] 0, T]$ and $u(0)=u_{0}$. More precisely, we will use Theorem 3.14 and Corollary 3.15 to obtain results of existence and uniqueness of solutions of the Cauchy problem $(73)$ in $W_{p}^{k}\left(\mathbb{R}^{n}, E\right)$ if $1 \leq p<\infty$ and in $B U C^{k}\left(\mathbb{R}^{n}, E\right)$ if $p=\infty$. In these cases the domain $D\left(A(t)_{\mathfrak{F}_{p}^{k}}\right)$ depends on $t$ due to Remark 3.9 and to Proposition 1.12 in [Gu93]. We know about $D\left(A(t)_{\mathfrak{F}_{p}^{k}}\right)$, according to (55) and (72), only that

$$
\begin{gathered}
\stackrel{\circ}{B}_{p, 1}^{k+m}\left(\mathbb{R}^{n}, E\right) \stackrel{d}{\hookrightarrow} D\left(A(\tau)_{W_{p}^{k}\left(\mathbb{R}^{n}, E\right)}\right) \stackrel{d}{\hookrightarrow} \stackrel{\circ}{B}_{p, \infty}^{k+m}\left(\mathbb{R}^{n}, E\right), \quad 1 \leq p<\infty, \\
b_{\infty, 1}^{k+m}\left(\mathbb{R}^{n}, E\right) \stackrel{d}{\hookrightarrow} D\left(A(\tau)_{B U C^{k}\left(\mathbb{R}^{n}, E\right)}\right) \stackrel{d}{\hookrightarrow} b_{\infty, \infty}^{k+m}\left(\mathbb{R}^{n}, E\right) .
\end{gathered}
$$

In this case we will apply the following theorem.

Proposition 4.1. Let $(A(t))_{t \in J}$ a family of linear operator in Banach space $\mathbb{E}_{0}$ with domain $\mathbb{E}_{1}(t):=D(A(t))$ for $t \in J$, such that:

a) $\mathbb{E}_{1}(t) \stackrel{d}{\hookrightarrow} \mathbb{E}_{0}$ for all $t \in J$. 
PSEUDODIFFERENTIAL OPERATORS ON VECTOR-VALUED SOBOLEV SPACES 31

b) There exists a constant $M \geq 1$, so that $\{z \in \mathbb{C}: \operatorname{Re}(z) \geq 0\} \subset \rho(-A(t))$ and $\left\|(\lambda I+A(t))^{-1}\right\|_{\mathcal{L}\left(\mathbb{E}_{0}\right)} \leq \frac{M}{1+|\lambda|}$ for all $\operatorname{Re}(\lambda) \geq 0$ and $t \in J$.

c) There are constants $\theta \in] 0,1[, q \in[1, \infty[, \kappa \geq 1$ and a Banach space $\mathbb{E}_{\theta, q}:=\left(\mathbb{E}_{\theta, q} ;\|\cdot\|_{\theta, q}\right)$ so that

$$
\mathbb{E}_{\theta, q}=\left(\mathbb{E}_{0}, \mathbb{E}_{1}(t)\right)_{\theta, q}=: \mathbb{E}_{\theta, q}(t)
$$

and

for all $x \in \mathbb{E}_{\theta, q}$ and $t \in J$.

$$
\kappa^{-1}\|x\|_{\theta, q} \leq\|x\|_{\mathbb{E}_{\theta, q}(t)} \leq \kappa\|x\|_{\theta, q}
$$

d) There exist constants $\rho \in] 1-\theta, 1\left[\right.$ and $\eta>0$ with $t \longmapsto A^{-1}(t) \in$ $C^{\rho}\left(J, \mathcal{L}\left(\mathbb{E}_{0}, \mathbb{E}_{\theta, q}\right)\right)$ and

$$
\sup _{t, t^{\prime} \in J, t \neq t^{\prime}}\left(\frac{\left\|A^{-1}(t)-A^{-1}\left(t^{\prime}\right)\right\|_{\mathcal{L}\left(\mathbb{E}_{0}, \mathbb{E}_{\theta, q}\right)}}{\left|t-t^{\prime}\right|^{\rho}} \leq \eta .\right.
$$

Moreover, let $u_{0} \in \mathbb{E}_{0}, F=\left(\mathbb{E}_{0}, \mathbb{E}_{\theta, q}\right)_{\widetilde{\theta}, r}$ for some $\left.\widetilde{\theta} \in\right] 0,1[, r \in[1, \infty[$ and let

$$
f \in C^{\sigma}\left(J, \mathbb{E}_{0}\right)+C(J, F)
$$

for some $\sigma \in] 0,1[$. Then the Cauchy problem

$$
\left\{\begin{aligned}
\partial_{t} u+A(t) u & =f(t), \quad t \in J \backslash\{0\}, \\
u(0) & =u_{0},
\end{aligned}\right.
$$

has a unique solution

$$
u \in C\left(J, \mathbb{E}_{0}\right) \cap C^{1}\left(J \backslash\{0\}, \mathbb{E}_{0}\right) .
$$

Proof. See [Am95], Chapter IV, Theorem 2.5.1.

Lemma 4.2. Let $\alpha, m \in \mathbb{R}$ with $0<\alpha<1$ and $m>0, p \in[1, \infty]$, $\mathcal{A}:=\{a(t, \cdot): t \in J\} \subset S_{1,0}^{m, \rho_{n}}\left(\mathbb{R}^{n}, \mathcal{L}\left(E_{0}\right)\right)$ a family of parabolic symbols with the same constants $\omega$ and $\kappa$, such that

$$
t \longmapsto a(t, \cdot) \in C^{\alpha}\left(J, S_{1,0}^{m, \rho_{n}}\left(\mathbb{R}^{n}, \mathcal{L}\left(E_{0}\right)\right)\right) .
$$

Furthermore let $k \in \mathbb{N}_{0}, \mathfrak{F}_{p}^{k}=W_{p}^{k}$ for $1 \leq p<\infty, \mathfrak{F}_{\infty}^{k}=B \cup C^{k}$ (in this case, $p=\infty$, we assume $m>k), \mathcal{B}$ as in (40) and $A(t):=\left.\widetilde{a(t, D)}\right|_{\mathcal{B}_{p}^{k+\infty}\left(\mathbb{R}^{n}, E_{0}\right)}$ for $t \in J$. Then there exists a constant $\widetilde{\omega}>0$ independent of $t$, such that the operator $\widetilde{A}(t):=\widetilde{\omega} I+A(t)_{\mathfrak{F}_{p}^{k}}, t \in J$, satisfies:

a) $\mathbb{E}_{1}(t):=D(\widetilde{A}(t)) \stackrel{d}{\hookrightarrow} \mathfrak{F}_{p}^{k}\left(\mathbb{R}^{n}, E_{0}\right):=\mathbb{E}_{0}$ for all $t \in J$.

b) There exists a constant $M \geq 1$ such that $\{z \in: \operatorname{Re}(z) \geq 0\} \subset \rho(-\widetilde{A}(t))$ and

$\left\|(\lambda I+\widetilde{A}(t))^{-1}\right\|_{\mathcal{L}\left(\mathbb{E}_{0}\right)} \leq \frac{M}{1+|\lambda|}$ for all $\operatorname{Re}(\lambda) \geq 0$ and $t \in J$. 
c) For each $p, q \in[1, \infty]$ and $\theta \in] 0,1[$ there exists a constant $\kappa>0$ such that

$$
\mathbb{E}_{\theta, q}:=B_{p, q}^{k+\theta m}\left(\mathbb{R}^{n}, E_{0}\right) \approx\left(\mathbb{E}_{0}, \mathbb{E}_{1}(t)\right)_{\theta, q} \quad \text { for all } t \in J
$$

and

$$
\kappa^{-1}\|x\|_{\theta, q} \leq\|x\|_{\left(\mathbb{E}_{0}, \mathbb{E}_{1}(t)\right)_{\theta, q}} \leq \kappa\|x\|_{\theta, q}
$$

for all $x \in\left(\mathbb{E}_{\theta, q} ;\|\cdot\|_{\theta, q}\right)$ and $t \in J$.

d) For each $\theta \in] 0,1[, p, q, r \in[1, \infty], \gamma \in] 0, \theta m\left[\right.$ and $\widetilde{\theta}:=\frac{\gamma}{m \theta}$ it holds

$$
B_{p, r}^{k+\gamma}\left(\mathbb{R}^{n}, E_{0}\right) \approx\left(\mathbb{E}_{0}, \mathbb{E}_{\theta, q}\right)_{\widetilde{\theta}, r} .
$$

e) For each $\theta \in] 0,1[$ and each $q \in[1, \infty]$ there is a constant $\eta>0$ such that

$$
t \longmapsto \widetilde{A}^{-1}(t) \in C^{\alpha}\left(J, \mathcal{L}\left(\mathbb{E}_{0}, \mathbb{E}_{\theta, q}\right)\right)
$$

and

$$
\sup _{t, t^{\prime} \in J, t \neq t^{\prime}}\left(\frac{\left\|\widetilde{A}^{-1}(t)-\widetilde{A}^{-1}\left(t^{\prime}\right)\right\|_{\mathcal{L}\left(\mathbb{E}_{0}, \mathbb{E}_{\theta, q}\right)}}{\left|t-t^{\prime}\right|^{\alpha}}\right) \leq \eta
$$

Proof. Due to Theorem 3.14 and Corollary 3.15 we get that there are constants $R>0$ and $\beta \in] \frac{\pi}{2}, \pi\left[\right.$, independent of $t$, such that $\sum_{\beta, R} \subset \rho\left(-A(t)_{\mathfrak{F}_{p}^{k}}\right)$ for all $t \in J$. We will prove for a fixed $\widetilde{\omega} \geq R$ that the operator $\widetilde{A}(t):=$ $\widetilde{\omega} I+A(t)_{\mathfrak{F}_{p}^{k}}, t \in J$, satisfies the assertions $\left.a\right)-e$ ). From Theorem 3.14, Corollary 3.15, (19), Lemma 2.1e) and (41) it follows $a$ ), $b$ ) and

$$
\begin{aligned}
& B_{p, 1}^{k+m}\left(\mathbb{R}^{n}, E_{0}\right) \hookrightarrow D\left(A(t)_{\mathfrak{F}_{p}^{k}}\right) \hookrightarrow B_{p, \infty}^{k+m}\left(\mathbb{R}^{n}, E_{0}\right), \\
& B_{p, 1}^{k}\left(\mathbb{R}^{n}, E_{0}\right) \hookrightarrow \mathfrak{F}_{p}^{k}\left(\mathbb{R}^{n}, E_{0}\right) \hookrightarrow B_{p, \infty}^{k}\left(\mathbb{R}^{n}, E_{0}\right)
\end{aligned}
$$

for all $t \in J$ and $p \in[1, \infty]$. From this and (18) we get for each $\theta \in] 0,1[$ and $p, q \in[1, \infty], t \in J$ that:

$$
\left(\mathfrak{F}_{p}^{k}\left(\mathbb{R}^{n}, E_{0}\right), D(\widetilde{A}(t))\right)_{\theta, q} \approx B_{p, q}^{k+\theta m}\left(\mathbb{R}^{n}, E_{0}\right),
$$

and it holds (76). Thus $\widetilde{A}$ satisfies the assertion $c$ ). The assertion $d$ ) follows from

$$
B_{p, 1}^{k} \hookrightarrow \mathfrak{F}_{p}^{k} \hookrightarrow B_{p, \infty}^{k}, \quad B_{p, 1}^{k+\theta m} \hookrightarrow B_{p, q}^{k+\theta m} \hookrightarrow B_{p, \infty}^{k+\theta m} \quad \forall p, q \in[1, \infty]
$$

and from (18), because

$\left(\mathbb{E}_{0}, \mathbb{E}_{\theta, q}\right)_{\widetilde{\theta}, r}=\left(\mathfrak{F}_{p}^{k}, B_{p, q}^{k+\theta m}\right)_{\widetilde{\theta}, r} \approx\left(B_{p, 1}^{k}, B_{p, 1}^{k+\theta m}\right)_{\widetilde{\theta}, r} \approx B_{p, r}^{(1-\widetilde{\theta}) k+\widetilde{\theta}(k+\theta m)}=B_{p, r}^{k+\gamma}$

for $\widetilde{\theta}=\frac{\gamma}{m \theta}$. Now we will show $e$ ). According to $\mathfrak{F}_{p}^{k}\left(\mathbb{R}^{n}, E_{0}\right) \hookrightarrow B_{p, \infty}^{k}\left(\mathbb{R}^{n}, E_{0}\right)$ for all $p \in[1, \infty]$ it holds that

$$
\left\|\widetilde{A}(t)^{-1}\right\|_{\mathcal{L}\left(\mathfrak{F}_{p}^{\left.k, B_{p, \infty}^{k+\theta m}\right)}\right.} \leq c_{1}\left\|\left(\widetilde{\omega} I+\left.\widetilde{a(t, D)}\right|_{\mathcal{B}_{p, \infty}^{k+m}}\right)^{-1}\right\|_{\mathcal{L}\left(B_{p, \infty}^{k}, B_{p, \infty}^{k+\theta m}\right)}
$$


for all $t \in J$, where the constant $c_{1}$ does not depend on $t$. Furthermore there are constants $c_{2}>0$ and $R>0$ independent of $t$ (due to Proposition 3.5 and $\left.B_{p, \infty}^{k+m}\left(\mathbb{R}^{n}, E_{0}\right) \hookrightarrow B_{p, \infty}^{k+\theta m}\left(\mathbb{R}^{n}, E_{0}\right)\right)$ such that

$$
\left\|\left(\lambda I+\left.\widetilde{a(t, D)}\right|_{\mathcal{B}_{p, \infty}^{k+m}}\right)^{-1}\right\|_{\mathcal{L}\left(B_{p, \infty}^{k}, B_{p, \infty}^{k+j m}\right)} \leq c_{2}
$$

for all $\lambda \in \Sigma_{\frac{\pi}{2}, R}, t \in J$ and $j=\theta, 1$. Now we take some $\widetilde{\omega} \geq R$ (thus $\widetilde{\omega} \in \Sigma_{\frac{\pi}{2}, R}$. Then we get for all $t, t^{\prime} \in J$ that

$$
\begin{aligned}
& \left\|\widetilde{A}(t)^{-1}-\widetilde{A}\left(t^{\prime}\right)^{-1}\right\|_{\mathcal{L}\left(\mathfrak{F}_{p}^{k}, B_{p, \infty}^{k+\theta m}\right)} \\
& \leq c_{1}\left\|\left(\widetilde{\omega} I+\left.\widetilde{a(t, D)}\right|_{\mathcal{B}_{p, \infty}^{k+m}}\right)^{-1}-\left(\widetilde{\omega} I+\left.\widetilde{a\left(t^{\prime}, D\right)}\right|_{\mathcal{B}_{p, \infty}^{k+m}}\right)^{-1}\right\|_{\mathcal{L}\left(B_{p, \infty}^{k}, B_{p, \infty}^{k, \theta m}\right)} \\
& \leq c_{1}\left\|\left(\widetilde{\omega} I+\left.\widetilde{a(t, D)}\right|_{\mathcal{B}_{p, \infty}^{k+m}}\right)^{-1}\right\|_{\mathcal{L}\left(B_{p, \infty}^{k}, B_{p, \infty}^{k+\theta m}\right)} \\
& \cdot|\widetilde{a(t, D)}|_{\mathcal{B}_{p, \infty}^{k+m}}-\left.\widetilde{a\left(t^{\prime}, D\right)}\right|_{\mathcal{B}_{p, \infty}^{k+m}} \|_{\mathcal{L}\left(B_{p, \infty}^{k+m}, B_{p, \infty}^{k}\right)} \\
& \cdot\left\|\left(\widetilde{\omega} I+\left.\widetilde{a\left(t^{\prime}, D\right)}\right|_{\mathcal{B}_{p, \infty}^{k+m}}\right)^{-1}\right\|_{\mathcal{L}\left(B_{p, \infty}^{k}, B_{p, \infty}^{k+m}\right)} \\
& \stackrel{(80)}{\leq} c_{1} c_{2}^{2}\left\|\left.\widetilde{a(t, D)}\right|_{\mathcal{B}_{p, \infty}^{k+m}}-\left.\widetilde{a\left(t^{\prime}, D\right)}\right|_{\mathcal{B}_{p, \infty}^{k+m}}\right\|_{\mathcal{L}\left(B_{p, \infty}^{k+m}, B_{p, \infty}^{k}\right)} \\
& \leq \eta_{\infty}\left|t-t^{\prime}\right|^{\alpha} \quad \text { (due to Corollary } 3.2 \text { and (74)). }
\end{aligned}
$$

There, $\eta_{\infty}:=c_{1} c_{2}^{2}\|a(\cdot, \cdot)\|_{C^{\alpha}}$ is a constant independent of $t$. Therefore, it holds (78) for $q=\infty$ and all $\theta \in] 0,1\left[\right.$. Now, let $\theta^{\prime} \in \mathbb{R}$ with $0<\theta<$ $\theta^{\prime}<1$. Then we get for all $q \in[1, \infty]$, according to Lemma 2.1c), that $B_{p, \infty}^{k+\theta^{\prime} m}\left(\mathbb{R}^{n}, E_{0}\right) \hookrightarrow B_{p, q}^{k+\theta m}\left(\mathbb{R}^{n}, E_{0}\right)$, and from this follows that

$$
\begin{array}{r}
\sup _{t, t^{\prime} \in J, t \neq t^{\prime}}\left(\frac{\left\|\widetilde{A}^{-1}(t)-\widetilde{A}^{-1}\left(t^{\prime}\right)\right\|_{\mathcal{L}\left(\mathbb{E}_{0}, \mathbb{E}_{\theta, q}\right)}}{\left|t-t^{\prime}\right|^{\alpha}}\right) \\
\quad=\sup _{t, t^{\prime} \in J, t \neq t^{\prime}}\left(\frac{\left\|\widetilde{A}^{-1}(t)-\widetilde{A}^{-1}\left(t^{\prime}\right)\right\|_{\mathcal{L}\left(\mathbb{E}_{0}, B_{p, q}^{k+\theta m}\right)}}{\left|t-t^{\prime}\right|^{\alpha}}\right) \\
\leq \sup _{t, t^{\prime} \in J, t \neq t^{\prime}}\left(\frac{c\left\|\widetilde{A}^{-1}(t)-\widetilde{A}^{-1}\left(t^{\prime}\right)\right\|_{\mathcal{L}\left(\mathbb{E}_{0}, B_{p, \infty}^{k+\theta^{\prime} m}\right)}}{\left|t-t^{\prime}\right|^{\alpha}}\right) \\
=c \sup _{t, t^{\prime} \in J, t \neq t^{\prime}}\left(\frac{\left\|\widetilde{A}^{-1}(t)-\widetilde{A}^{-1}\left(t^{\prime}\right)\right\|_{\mathcal{L}\left(\mathbb{E}_{0}, \mathbb{E}_{\theta^{\prime}, \infty}\right)}}{\left|t-t^{\prime}\right|^{\alpha}}\right)
\end{array}
$$




$$
\leq c \eta_{\infty}=: \eta
$$

Thus the assertion $e$ ) follows.

Theorem 4.3. Let $\alpha, m, T \in \mathbb{R}^{+}$with $\left.0<\alpha<1, p \in[1, \infty], \theta \in\right] 1-\alpha, 1[$, $\rho_{n}$ as in $(3), J:=[0, T], \mathcal{A}:=\{a(t, \cdot): t \in J\} \subset S_{1,0}^{m, \rho_{n}}\left(\mathbb{R}^{n}, \mathcal{L}\left(E_{0}\right)\right)$ a family of parabolic symbols with the same constants $\omega$ and $\kappa$, so that

$$
t \longmapsto a(t, \cdot) \in C^{\alpha}\left(J, S_{1,0}^{m, \rho_{n}}\left(\mathbb{R}^{n}, \mathcal{L}\left(E_{0}\right)\right)\right) .
$$

Furthermore, let $k \in \mathbb{N}_{0}, \mathfrak{F}_{p}^{k}=W_{p}^{k}$ for $1 \leq p<\infty, \mathfrak{F}_{\infty}^{k}=B U C^{k}$ (in this case, $p=\infty, m>k$ is assumed), $\mathcal{B}$ as in (40) and $A(t):=\left.\widetilde{a(t, D)}\right|_{\mathcal{B}_{p, \infty}^{k+m}\left(\mathbb{R}^{n}, E_{0}\right)}$ for $t \in J$. If $u_{0} \in \mathfrak{F}_{p}^{k}\left(\mathbb{R}^{n}, E_{0}\right)$ and

$$
f \in C^{\sigma}\left(J, \mathfrak{F}_{p}^{k}\left(\mathbb{R}^{n}, E_{0}\right)\right)+C\left(J, B_{p, r}^{k+\gamma}\left(\mathbb{R}^{n}, E_{0}\right)\right)
$$

for some $\sigma \in] 0,1[, r \in[1, \infty[$ and some $\gamma \in] 0, \theta m[$, then the Cauchy problem

$$
\left\{\begin{array}{rl}
\partial_{t} u+A(t)_{\mathfrak{F}_{p}^{k}} & u=f(t), \quad t \in J \backslash\{0\}, \\
u(0) & =u_{0},
\end{array}\right.
$$

has a unique solution

$$
u \in C\left(J, \mathfrak{F}_{p}^{k}\left(\mathbb{R}^{n}, E_{0}\right)\right) \cap C^{1}\left(J \backslash\{0\}, \mathfrak{F}_{p}^{k}\left(\mathbb{R}^{n}, E_{0}\right)\right) .
$$

Proof. It follows from Proposition 4.1 and Lemma 4.2: The unique solution of $(81)$ is $u(t):=e^{\widetilde{\omega} t} v(t)$, where

$$
v \in C\left(J, \mathfrak{F}_{p}^{k}\left(\mathbb{R}^{n}, E_{0}\right)\right) \cap C^{1}\left(J \backslash\{0\}, \mathfrak{F}_{p}^{k}\left(\mathbb{R}^{n}, E_{0}\right)\right)
$$

is the unique solution of the problem

$$
\left\{\begin{array}{c}
\partial_{t} u+\widetilde{A}(t) u=e^{-\widetilde{\omega} t} f(t), \quad t \in J \backslash\{0\}, \\
u(0)=u_{0},
\end{array}\right.
$$

with $\widetilde{A}(t)$ as in Lemma 4.2 .

Remark 4.4. Let conditions in Theorem 3.14 be satisfied. If $1 \leq p<\infty$, $-A(\tau)_{W_{p}^{k}}$ generates a $C_{0}$-semigroup on $W_{p}^{k}\left(\mathbb{R}^{n}, E_{0}\right)$ with

$$
\left\|\left(\lambda+A(\tau)_{W_{p}^{k}}\right)^{-1}\right\|_{\mathcal{L}\left(W_{p}^{k}\right)} \leq \frac{M_{1}}{1+|\lambda|}
$$

for all $\lambda \in \Sigma_{\frac{\pi}{2}, R}$ and $\tau \in \mathfrak{I}$. Doing

$$
\begin{aligned}
C_{14}:=\frac{4 \rho_{n} \omega_{n} n^{\frac{n+3}{2}} \kappa^{n+1}}{(2 \pi)^{n}}\left(\sum_{|\alpha|=n+1} 1\right) \max _{|\alpha| \leq \rho_{n}}\left\{\sum_{j=1}^{|\alpha|} \sum_{\substack{\alpha_{1}+\cdots+\alpha_{j} \\
\alpha_{1}, \ldots, \alpha_{j} \neq 0}} 1\right\} \\
\cdot \|\left.\sum_{i=0}^{1} \frac{|\cdot| \theta_{i}-n}{1+|\cdot|}\right|_{L_{1}} \max _{|\alpha| \leq n+1}\left\{C_{11} \sum_{\gamma \leq \alpha}\left(\begin{array}{c}
\alpha \\
\gamma
\end{array}\right)\right\} \\
\cdot \max \left\{\frac{2 m}{m^{2}-\theta_{0}^{2}}, \frac{2 m}{m^{2}-\left(1-\theta_{1}\right)^{2}}, \frac{1}{n-\theta_{0}}+\frac{1}{n}\right\}
\end{aligned}
$$


it holds that $M_{1}$ in Proposition 3.13 satiesfies

$$
M_{1}=C_{14}\left(\sup _{\tau \in \mathfrak{I}}\left\|a_{\tau}\right\|_{S_{1,0}^{m, \rho_{n}}}\right)^{n} .
$$

If we consider that $\left\{a_{\tau}: \tau \in \mathfrak{I}\right\} \subset \bar{B}_{\sqrt[n]{C_{14}^{-1}}}\left(0_{S_{1,0}^{m}, \rho_{n}}\right)$, then $M_{1} \leq 1$ and therefore the family $\left\{A(\tau)_{W_{p}^{k}}: \tau \in \mathfrak{I}\right\}$ is stable (see [Ka93]). Now, let $Y:=$ $\stackrel{\circ}{B}_{p, 1}^{k+m}\left(\mathbb{R}^{n}, E_{1}\right)$ and $X:=W_{p}^{k}\left(\mathbb{R}^{n}, E_{0}\right)$ for $1 \leq p<\infty$. For $\lambda \in \mathbb{R}$ with $\lambda>R$ we get

$$
\begin{gathered}
Y \stackrel{d}{\hookrightarrow} X, \\
\left(\lambda+A(\tau)_{W_{p}^{k}}\right)^{-1}(Y)=\left(\lambda+\left.A(\tau)\right|_{\stackrel{B}{p}, 1_{p}^{k+m}}\right)^{-1}(Y)=\stackrel{\circ}{B}_{p, 1}^{k+2 m} \stackrel{d}{\hookrightarrow} \stackrel{\circ}{B}_{p, 1}^{k+m}=Y
\end{gathered}
$$

and (due to Proposition 3.5)

$$
\begin{aligned}
\left\|\left(\lambda+A(\tau)_{W_{p}^{k}}\right)^{-k}\right\|_{\mathcal{L}(Y)} & \leq \frac{C_{6}^{k}}{(1+\lambda)^{k}} \\
& \leq \frac{C_{6}^{k}}{(\lambda-R)^{k}}
\end{aligned}
$$

for all $\lambda>R$ and $k=1,2, \ldots$, where

$$
C_{6}=\underbrace{C_{3} \kappa^{n+1}\left(\max _{|\alpha| \leq \rho_{n}}\left\{\sum_{\substack{j=1 \\ \alpha_{1}+\cdots,+\alpha_{j}=\alpha \\ \alpha_{1}, \ldots, \alpha_{j} \neq 0}}^{|\alpha|} 1\right\}\right)}_{=: C_{15}}\left(\sup _{\tau \in \mathfrak{I}}\left\|a_{\tau}\right\|_{S_{1,0}^{m, \rho_{n}}}\right)^{n}
$$

If $\left\{a_{\tau}: \tau \in \mathfrak{I}\right\} \subset \bar{B}_{\sqrt[n]{C_{15}^{-1}}}\left(0_{S_{1,0}^{m, \rho_{n}}}\right)$, then it follows from (84) that $Y$ is admissible with respect to $\left\{A(\tau)_{W_{p}^{k}}: \tau \in \mathfrak{I}\right\}$ (see Definition 21 and Proposition 2.3 in [Ka70]). These Remarks will be useful for the analysis, in an forthcoming paper, of the semilinear pseudo differential equation

$$
\left\{\begin{array}{l}
\partial_{t} u+a(t, D) u=f(t, u), \quad 0<t \leq T, \\
u(0)=u_{0} .
\end{array}\right.
$$

on the Sobolev space $W_{p}^{k}\left(\mathbb{R}^{n}, E\right), 1 \leq p<\infty$.

\section{REFERENCES}

[Am95] H. Amann, Linear and Quasilinear Parabolic Problems, Volumen I, Birkhäuser Verlag, 1995.

[Am97] H. Amann, Operator-Valued Fourier Multipliers, Vector-Valued Besov Spaces, and Applications, Math. Nachr. 186 (1997), 5-56.

[Am00] H. Amann Coagulation-Fragmentation Processes, Arch. Rot. Mech. Anal. 151 (2000), 339-366.

[Am01] H. Amann, Elliptic Operators with Infinite Dimensional State Spaces, J. Evo. Equ. 1 (2001), 143-188.

[Am03] H. Amann, Vector-Valued Distributions and Fourier Multipliers, Zürich 2003, Download from http://www.math.unizh.ch/amann/files/distributions.pdf.

[Ba09] B. Barraza Martínez, Pseudodifferentialoperatoren mit nichtregulären banachraumwertigen Symbolen, Dissertation, Johannes Gutenberg-Universität Mainz, 2009. 
[BL76] J. Berg, and J. Löfstrom, Interpolation Spaces, Springer,1976.

[Bo87] J. Bourgaing, Vector-valued Hausdorff-Young Inequalities and Applications, in Geometric Aspects of Functional Analisis (1986/87), 239-247.

[GW03] M. Girardi and L. Weis, Operator-Valued Fourier Multiplier Theorems on Besov Spaces, Math. Nachr. 251 (2003), 34-51.

[Gu93] D. Guidotti, On Elliptic Systems in $L^{1}$, Osaka J. Math., 30 (1993), 397-429.

[Ka70] T. Kato, Linear evolution equations of "hyperbolic" type, J. Fac. Sci. Univ. Tokyo, Sect. I, 17 (1970), 241-258.

[Ka93] T. Kato, Abstract evolution equations, linear and quasilinear, revisited, In $\mathrm{H}$. Komatsu, editor, "Functional Analysis and Related Topics", Lecture Notes in Math., Springer (1993), 103-125.

[Ki01] Chr. Kiehn, Analytic Semigroup of Pseudodifferential Operators on $L_{p}\left(\mathbb{R}^{n}, E\right)$, Preprint Reihe des Fachbereichs Mathematik Nr 10, Johannes GutenbergUniversität, 2001.

[Ki03] Chr. Kiehn, Analytic Semigroups of Pseudodifferential Operators on Vector Valued Function Spaces, Shaker Verlag, 2003.

[Ku81] H. Kumano-go, Pseudodifferential Operators, MIT Press, Cambridge, MA,1981.

[LLM98] F. Lancien, G. Lancien and Ch. Le Merdy, A Joint Functional Calculus for Sectorial Operators with Commuting Resolvents, Proc. London Math. Soc. (3) 77 (1998), 387-414.

[Lu95] A. Lunardi, Analytic Semigroups and Optimal Regularity in Parabolic Problems, Birkhäuser Verlag, Boston 1995.

[NNH02] A. Noll, R. Haller, and H. Heck, Mikhlin's Theorem for Operator-Valued Multipliers in $n$ Variables, Math. Nach., 244 (2002), 110-130.

[PS06] P. Portal and Ž. Štrkalj, Pseudodifferential operators on Bochner Spaces and an application, Math. Z., 253 (2006), 805-819.

[Sc86] H. Schmeisser, Vector- Valued Sobolev and Besov Spaces, Seminar Analysis of the Karl-Weierstraß-Institute (1986), Band 96.

[We01] L. Weis, Operator-Valued Fourier Multiplier Theorems and Maximal $L_{p^{-}}$ Regularity, Math. Ann. 319 (2001), 735-758. 